\title{
Neutrophil-derived S100 calcium-binding proteins A8/A9 promote reticulated thrombocytosis and atherogenesis in diabetes
}

\author{
Michael J. Kraakman, ${ }^{1}$ Man K.S. Lee, ${ }^{1}$ Annas Al-Sharea, ${ }^{1}$ Dragana Dragoljevic, ${ }^{1}$ Tessa J. Barrett, ${ }^{2}$ Emilie Montenont, ${ }^{2,3}$ \\ Debapriya Basu, ${ }^{4}$ Sarah Heywood, ${ }^{1}$ Helene L. Kammoun, ${ }^{1}$ Michelle Flynn, ${ }^{1}$ Alexandra Whillas, ${ }^{1}$ Nordin M.J. Hanssen, ${ }^{1,5}$ \\ Mark A. Febbraio, ${ }^{6}$ Erik Westein, ${ }^{7}$ Edward A. Fisher, ${ }^{2}$ Jaye Chin-Dusting, ${ }^{8}$ Mark E. Cooper, ${ }^{9}$ Jeffrey S. Berger, ${ }^{2,3}$ Ira J. Goldberg, ${ }^{4}$ \\ Prabhakara R. Nagareddy, ${ }^{10}$ and Andrew J. Murphy ${ }^{1,11}$ \\ ${ }^{1}$ Haematopoiesis and Leukocyte Biology, Baker Heart and Diabetes Institute, Melbourne, Victoria, Australia. ${ }^{2}$ Division of Cardiology, ${ }^{3}$ Division of Hematology, and ${ }^{4}$ Division of Endocrinology, Diabetes \\ and Metabolism, New York University School of Medicine, New York, New York, USA. ㄹDepartment of Internal Medicine, Cardiovascular Research Institute Maastricht (CARIM), School of Cardiovascular \\ Diseases, Maastricht University, Maastricht, Netherlands. ${ }^{6}$ Cellular and Molecular Metabolism Laboratory, Garvan Institute of Medical Research, Darlinghurst, New South Wales, Australia. ${ }^{7}$ Vascular \\ Biomechanics, Baker Heart and Diabetes Institute, Melbourne, Victoria, Australia. ${ }^{8}$ Department of Pharmacology, Monash University, Clayton, Victoria, Australia. ${ }^{9}$ Diabetic Complications, Baker Heart \\ and Diabetes Institute, Melbourne, Victoria, Australia. ${ }^{10}$ Department of Nutrition Sciences, University of Alabama at Birmingham, Birmingham, Alabama, USA. ${ }^{11}$ Department of Immunology, Monash \\ University, Melbourne, Victoria, Australia.
}

\begin{abstract}
Platelets play a critical role in atherogenesis and thrombosis-mediated myocardial ischemia, processes that are accelerated in diabetes. Whether hyperglycemia promotes platelet production and whether enhanced platelet production contributes to enhanced atherothrombosis remains unknown. Here we found that in response to hyperglycemia, neutrophil-derived S100 calcium-binding proteins A8/A9 (S100A8/A9) interact with the receptor for advanced glycation end products (RACE) on hepatic Kupffer cells, resulting in increased production of IL-6, a pleiotropic cytokine that is implicated in inflammatory thrombocytosis. IL-6 acts on hepatocytes to enhance the production of thrombopoietin, which in turn interacts with its cognate receptor c-MPL on megakaryocytes and bone marrow progenitor cells to promote their expansion and proliferation, resulting in reticulated thrombocytosis. Lowering blood glucose using a sodium-glucose cotransporter 2 inhibitor (dapagliflozin), depleting neutrophils or Kupffer cells, or inhibiting S100A8/A9 binding to RACE (using paquinimod), all reduced diabetes-induced thrombocytosis. Inhibiting S100A8/A9 also decreased atherogenesis in diabetic mice. Finally, we found that patients with type 2 diabetes have reticulated thrombocytosis that correlates with glycated hemoglobin as well as increased plasma S100A8/A9 levels. These studies provide insights into the mechanisms that regulate platelet production and may aid in the development of strategies to improve on current antiplatelet therapies and to reduce cardiovascular disease risk in diabetes.
\end{abstract}

\section{Introduction}

Diabetes is a major risk factor for cardiovascular disease (CVD), which remains the leading cause of mortality worldwide (1-3). Atherosclerosis is the major underlying cause of CVD and is accelerated in people with diabetes $(4,5)$. How hyperglycemia accelerates atherogenesis and ultimately the downstream macrovascular complications seen in diabetes remains unresolved. Diabetes is associated with an expansion and activation of myeloid cells, par-

\section{Related Commentary: p. 2040}

Authorship note: M.J. Kraakman and M.K.S. Lee contributed equally to this work. P.R. Nagareddy and A.J. Murphy are co-senior authors.

Conflict of interest: M.E. Cooper has been on advisory boards of and received lecturer fees from AstraZeneca, Boehringer Ingelheim, and Eli Lilly in relation to SCLT2i's.

Submitted: December 23, 2016; Accepted: February 16, 2017

Reference information: / Clin Invest. 2017;127(6):2133-2147.

https://doi.org/10.1172/JC192450. ticularly monocytes and neutrophils, and impedes the resolution of atherosclerosis even when lipid levels are controlled (6). People with diabetes have been reported to also have significantly elevated platelet counts, which correlates with CVD (7). Platelets are small anucleated cells with a critical role in coagulation and have been causally implicated in the formation and progression of the atherosclerotic lesion along with forming thrombi and contributing specifically to CVD mortality (8-11). Importantly, a large meta-analysis with combined data from over 140 randomized trials has revealed that inhibiting platelet activation significantly reduces the risk of vascular events (12). However, people with diabetes are not afforded equal benefit with respect to reduction in CVD risk, with antiplatelet therapies being generally less efficacious in this population (13-15). This is considered to be at least in part due to enhanced platelet turnover as reflected by the increase in mean platelet volume (MPV) in diabetic versus nondiabetic subjects $(16,17)$. An increase in MPV signifies younger and more reactive platelets known as reticulated platelets $(14,16,18-22)$, which 
A

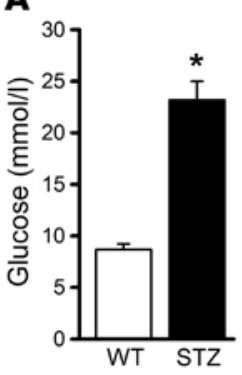

B

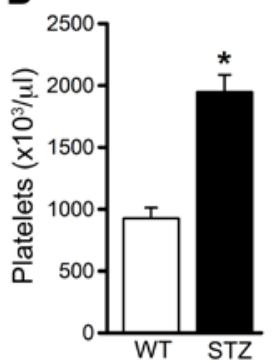

C

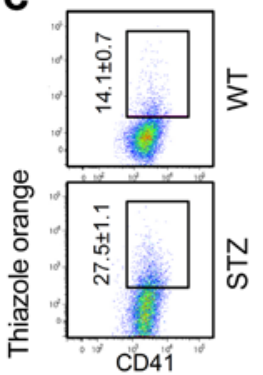

$\mathbf{F}$

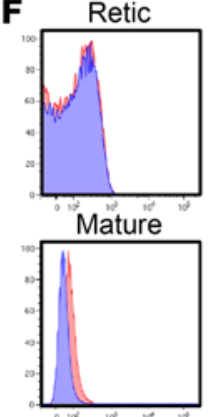

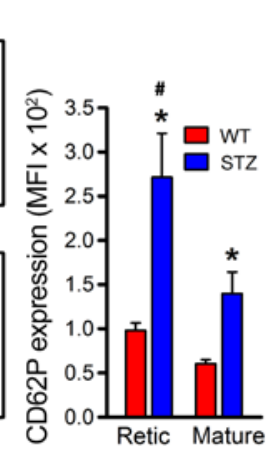

E Retic

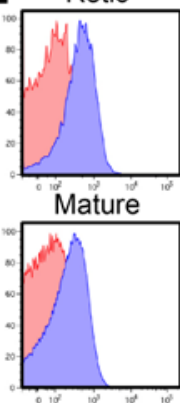

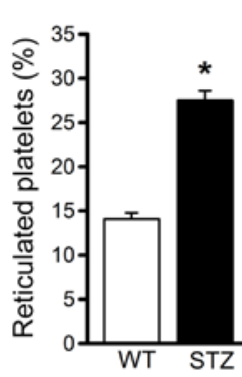

G

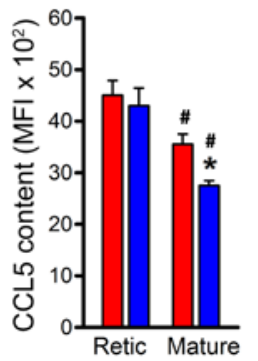

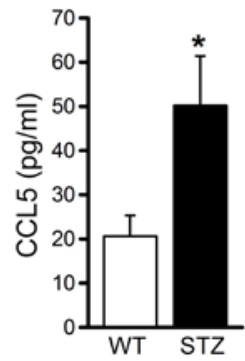

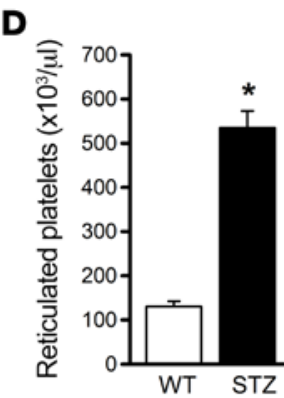

H

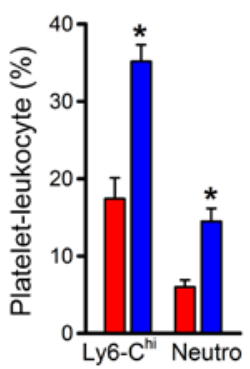

I

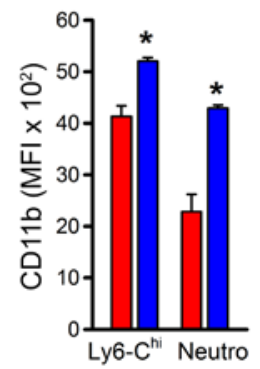

Figure 1. Reticulated thrombocytosis in diabetes. C57BL/6 mice were made diabetic with STZ and assessed after 4 weeks. (A) Blood glucose. (B) Circulating platelet levels from complete blood counts. (C and D) Abundance of circulating reticulated platelets expressed as percentage of platelets of total counts. (E-I) Markers of platelet activation: (E and F) Reticulated and mature platelet activation was measured by flow cytometry. (E) CD62P surface expression. (F) Intraplatelet CCL5 levels. (G) Plasma CCL5 levels were quantified by ELISA. (H and I) Platelet leukocyte aggregates (H) and leukocyte activation (CD11b levels) (I) were quantified by flow cytometry. $n=6$ control and 8 diabetic mice. ${ }^{*} P<0.05$ vs. WT for all graphs, except $\mathbf{E}$ and $\mathbf{F}$, where ${ }^{\#} P<0.05$ for cell effect and ${ }^{*} P<0.05$ for disease effect. All data represent the mean \pm SEM. $P$ values were obtained using a $t$ test, except in $\mathbf{E}$ and $\mathbf{F}$, where 2-way ANOVA was used.

display some resistance to standard antiplatelet therapies $(15,18$, 23). Moreover, immature platelet counts are gaining traction as an important additional measure of antiplatelet response independent of traditional risk factors $(24,25)$.

There is a significant role for reticulated platelets and enhanced platelet production, not only in thrombosis, but also in promoting atherogenesis $(8,10)$. The mechanisms responsible for increased levels of reticulated platelets and/or platelet turnover in diabetes are unknown. Reticulated platelets are newly formed thrombocytes produced from the fragmentation of long cytoplasmic protrusions from large polyploid cells called megakaryocytes (26). Reticulated thrombocytosis occurs in individuals with elevated thrombopoiesis reflecting increased megakaryocyte activity. Thrombopoiesis is regulated primarily by the hormone thrombopoietin (TPO), which binds to its cognate receptor c-MPL on several bone marrow (BM) progenitor cells and megakaryocytes (27). While TPO is constitutively produced from $\mathrm{BM}$ stromal cells and from the kidney, the predominant source of TPO is the liver (28). During periods of inflammation, hepatic TPO production is increased (29) through the actions of the pleiotropic cytokine interleukin-6 (IL-6) as part of the acute-phase response. This, in turn, promotes reticulated thrombocytosis $(30,31)$. Thrombocytosis can also occur through increased responsiveness to TPO, mediated by a failure in feedback pathways of c-MPL $(10,32)$ or, in the case of myeloproliferative neoplasms, activating mutations $(33,34)$.

It is proposed that understanding the mechanisms that contribute to reticulated thrombocytosis in diabetes $(16,21,35)$ will open new avenues to improve the efficacy of standard antiplatelet therapies and ultimately will reduce overall CVD risk. Therefore, we sought to investigate the pathways that contribute to enhanced platelet production in diabetes with the hope of identifying targetable pathways for subsequent intervention.

\section{Results}

Diabetes promotes reticulated thrombocytosis. To determine whether diabetes promotes thrombocytosis, we induced diabetes in C57BL/6 WT mice with streptozotocin (STZ) and observed them for 4 weeks (Figure 1A). Compared with nondiabetic WT control mice, diabetic mice had significantly elevated numbers of platelets in whole blood (Figure 1B). This appeared to be due to increased production, since both the percentage and the overall abundance of immature reticulated platelets were increased (Figure 1, C and D). We next examined some basic markers of platelet activation in both reticulated and mature platelets. We identified that reticulated platelets expressed more P-selectin (CD62P) on the surface than mature platelets in both WT and diabetic mice, suggesting that the immature platelets are more reactive than the matured platelets. Interestingly, both platelet subsets expressed higher levels of P-selectin in diabetic mice compared to WT, indicating that they are circulating in an activated state (Figure 1E). We also examined the intraplatelet content of the potent chemokine CCL5 and, somewhat surprisingly, found equal levels in the reticulated platelets from WT and diabetic mice, suggesting no changes in this chemokine when platelets are being produced. However, as the platelets matured, CCL5 levels were significantly reduced, an effect that was greater in the diabetic mice, suggesting enhanced 

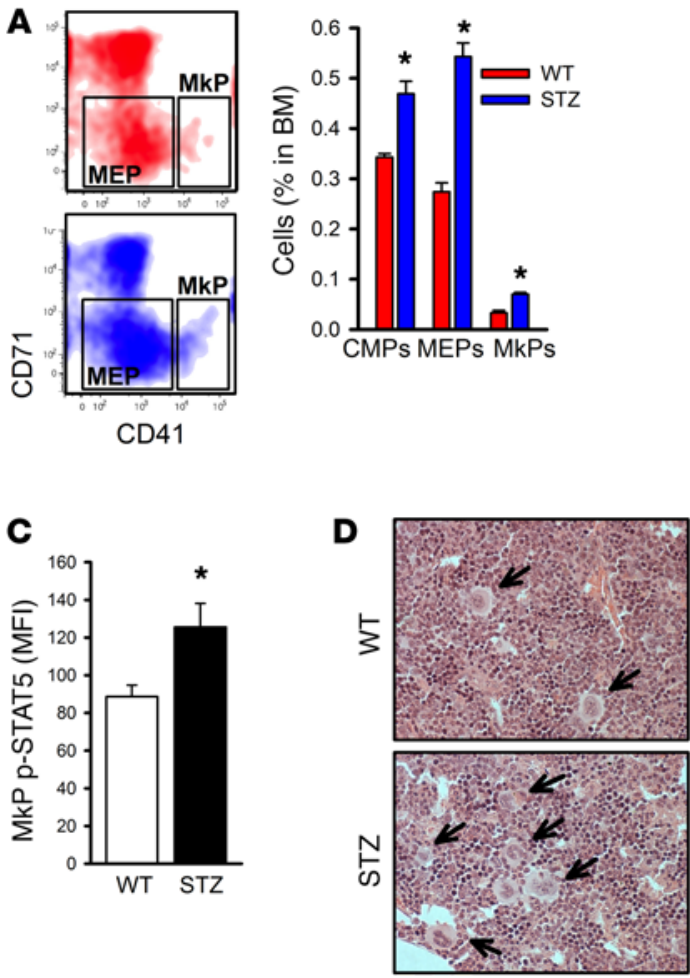
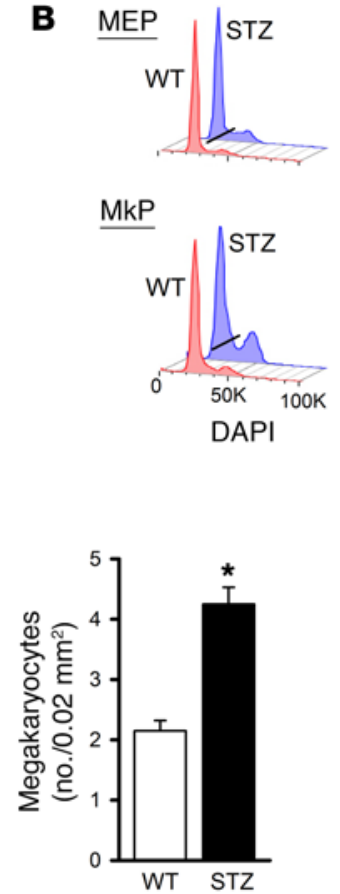

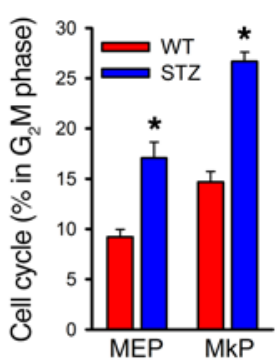

E

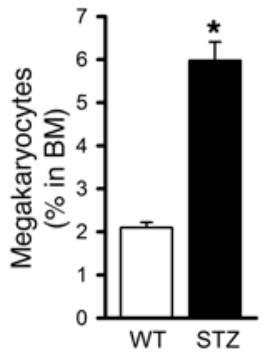

Figure 2. Reticulated thrombocytosis in diabetes is due to enhanced thrombopoiesis. C57BL/6 mice were made diabetic with STZ and assessed after 4 weeks. (A-C) The abundance (A) and proliferation (B) of BM progenitor cells and STAT5 phosphorylation in MkPs (C) were assessed by flow cytometry. (D) Bones were sectioned and stained with $\mathrm{H} \& \mathrm{E}$, and megakaryocytes were quantified as cells per field (original magnification, $\times 20$ ). (E) BM megakaryocytes were also quantified by flow cytometry. $n=6$ control and $n$ $=8$ diabetic. ${ }^{*} P<0.05$ vs. WT; all data represent the mean \pm SEM. $P$ values were obtained using a $t$ test. shedding/release (Figure 1F). Consequently, we observed higher plasma levels of CCL5 in the diabetic mice (Figure 1G). The increased abundance of these markers promoted more interactions with circulating Ly6- $\mathrm{C}^{\text {hi }}$ monocytes and neutrophils (Figure $1 \mathrm{H})$, thereby increasing the overall activation status of these leukocytes as assessed by CD11b expression (Figure 1I).

Reticulated thrombocytosis is caused by an expansion of BM progenitor cells. To investigate whether the increased levels of circulating reticulated platelets were due to enhanced production from the BM, we quantified BM progenitor cells within the lineage responsible for platelet production by flow cytometry. Diabetes induced an overall expansion of BM progenitor cells with a higher percentage of common myeloid progenitor cells (CMPs), megakaryocyte-erythrocyte progenitor cells (MEPs), and megakaryocyte progenitor cells (MkPs) in the $\mathrm{BM}$ of diabetic mice (Figure 2A). As we have shown previously, the percentage of the upstream self-renewing BM hematopoietic stem and multipotential progenitor cells (HSPCs) was not different between the WT and diabetic mice (ref. 6 and data not shown). Cell cycle analysis revealed that a greater percentage of the MEPs and MkPs were in the $G_{2} M$ phase, indicating that these cells were proliferating at a significantly greater rate (Figure $2 \mathrm{~B}$ ). We also observed more phosphorylated STAT5 in the MkPs, which is consistent with enhanced proliferative signaling via the TPO receptor c-MPL in these cells (ref. 36 and Figure 2C). Histological assessment also revealed the formation of more megakaryocytes in the $\mathrm{BM}$ of diabetic mice (Figure 2D), which was confirmed by flow cytometry (Figure $2 \mathrm{E}$ ). Taken together, these data confirm that the diabetic state is associated with increased production/proliferation of platelet progenitors resulting in elevated circulating levels of highly activated reticulated platelets.
Kupffer cells drive diabetes-associated thrombocytosis. As platelet homeostasis is controlled primarily by the hormone TPO, we examined whether diabetes-associated thrombocytosis was due to altered sensitivity to circulating TPO or increased circulating TPO levels. We measured the abundance of the TPO receptor (c-MPL) on BM progenitor cells and found no difference in c-MPL expression between WT and diabetic mice on either the MEPs or MkPs within the BM (Figure 3A). Platelets also express c-MPL, and upon TPO binding, a receptor/ligand internalization process is initiated, leading to reduced plasma TPO levels and an attenuation in platelet production. Thus, we next tested whether there was a reduction in c-MPL expression on circulating platelets, which could also explain an altered sensitivity to circulating TPO, but again no difference was seen (Figure 3B). There was, however, a significant increase in plasma TPO levels in diabetic mice compared with control mice (Figure 3C). These data suggest that thrombocytosis in diabetes is most likely mediated via the direct effects of TPO on its receptor. As TPO is produced primarily from the liver and elevated in times of stress, we decided to examine the liver. We first examined the abundance of Kupffer cells in the liver and found that diabetes was associated with a significant increase in the number of Kupffer cells (Figure 3D). We then assessed Kupffer cell-specific expression of IL-6, a known TPO-promoting cytokine. Kupffer cells from diabetic mice expressed more IL-6 than Kupffer cells from nondiabetic mice (Figure 3E). To support the hypothesis that Kupffer cells are involved in diabetes-associated thrombocytosis, we performed a Kupffer cell depletion study using a well-established protocol employing clodronate liposomes (37). As expected, the i.v. injection of clodronate liposomes resulted in a thorough depletion of liver Kupffer cells in both control and diabetic mice compared with mice injected with PBS-containing 
A

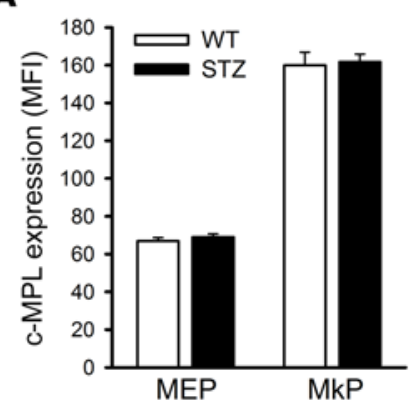

B

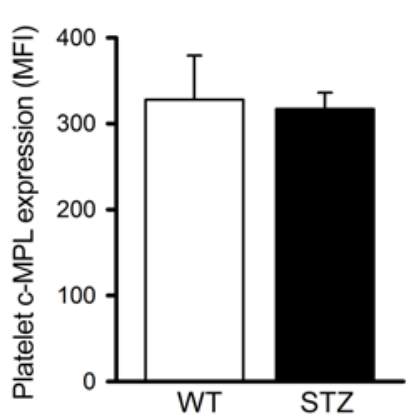

C

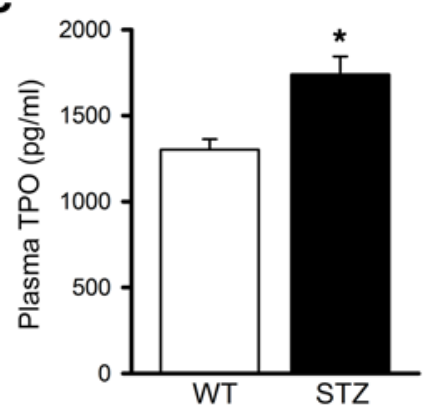

D

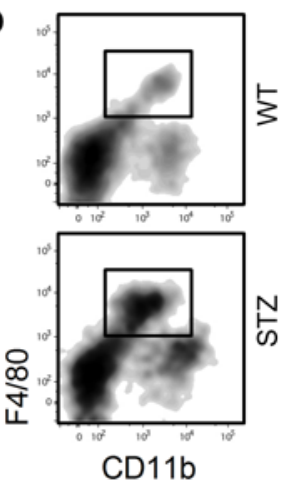

$\mathbf{F}$

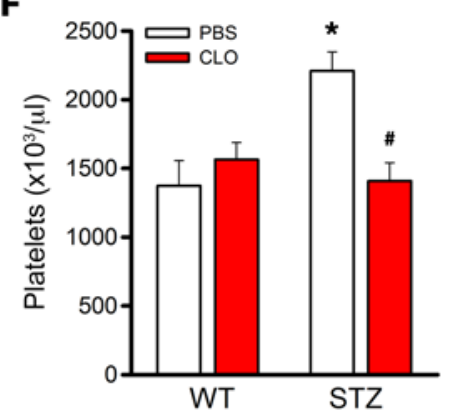

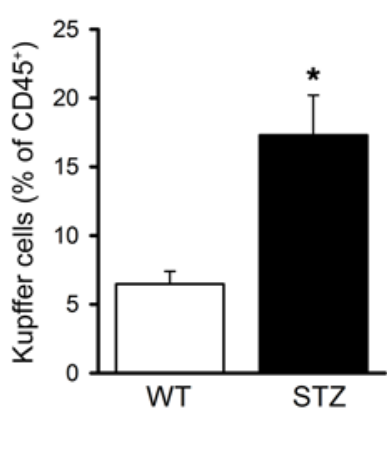

G

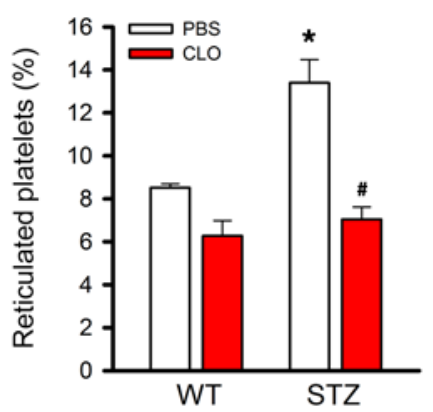

E

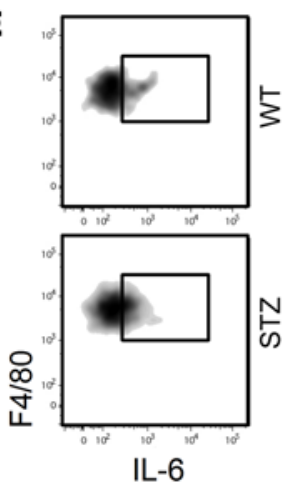

H

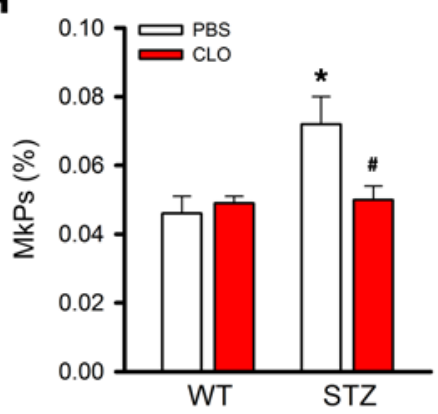

Figure 3. Liver phenotype in diabetic mice. (A-E) C57BL/6 mice were made diabetic with STZ and assessed after 4 weeks. C-MPL surface expression was measured on BM progenitors (A) and circulating platelets (B) by flow cytometry. (C) Plasma TPO was measured by ELISA. (D and E) Abundance of Kupffer cells (D) and Kupffer cell IL-6 expression (E) were quantified by flow cytometry. $n=6$ per group. ${ }^{*} P<0.05$. (F-H) WT and diabetic mice were treated with PBS or clodronate (CLO) liposomes $(250 \mu \mathrm{l} /$ mouse/i.v. injection) at day 1 and day 3 to deplete Kupffer cells. Mice were analyzed at day 7. Total (F) and percentage (G) of reticulated platelets. (H) Percentage of BM MkPs was quantified by flow cytometry. $n=5$ per group. ${ }^{*} P<0.05$ for disease effect, ${ }^{\#} P<0.05$ for treatment effect. All data represent the mean \pm SEM. See also Supplemental Figure 2. $P$ values were obtained using a $t$ test.

liposomes (Supplemental Figure 1, A and B; supplemental material available online with this article; https://doi.org/10.1172/ JCI92450DS1). The depletion of Kupffer cells in diabetic mice significantly decreased the number of circulating platelets and reticulated platelets as well as the percentage of MkPs in the BM (Figure 3, F-H). Kupffer cell depletion in nondiabetic mice did not alter these parameters (Figure 3, F-H). These data suggest that hepatic inflammation in the setting of diabetes is characterized by increased expression of IL- 6 in Kupffer cells, which plays an important role in diabetes-associated thrombocytosis.

Kupffer cell-derived IL-6 drives thrombocytosis in diabetes. To formally test the hypothesis that Kupffer cell-derived IL-6 drives diabetes-associated thrombocytosis, we performed a BM transplantation (BMT) study. WT mice were engrafted with BM from either WT or IL-6-deficient mice (Il6/-- mice) and allowed to reconstitute for 5 weeks before being made diabetic with STZ (Figure 4, A and B). After BMT, the newly recruited monocytes quickly seed the liver and mature to form Kupffer cells with gene expression signatures identical to those of the original Kupffer cell population (38). Further, we consciously chose to make the mice diabetic after reconstitution such that any newly recruited Kupffer cells, which we previously showed to be increased in diabetes (Figure 3D), would be of BM origin and thus IL-6 deficient (39). Consistent with earlier data, liver Kupffer cells were increased in diabetic mice, but the loss of IL-6 in hematopoietic cells did not influence Kupffer cell numbers in either the diabetic or the nondiabetic livers (Figure 4C). This finding is significant as alterations in thrombocytosis due to the loss of IL-6 occur downstream of liver Kupffer cells. Next, we measured the number of reticulated platelets and found a significant reduction in this parameter in 
A Donors:
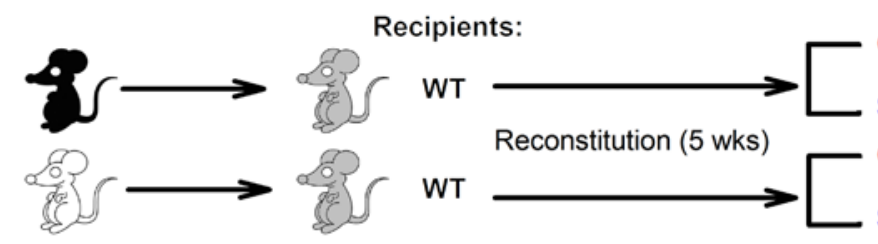

Control

1. WT

2. $116^{\circ /-}$

c

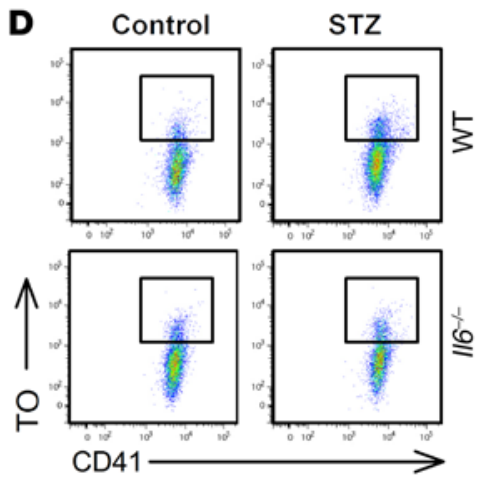

STZ Diabetes 4 wks Control STZ

B

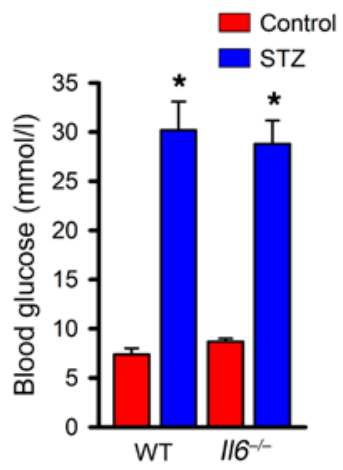

E

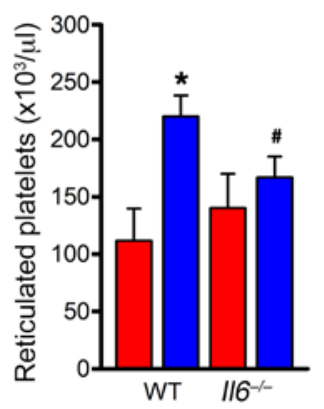

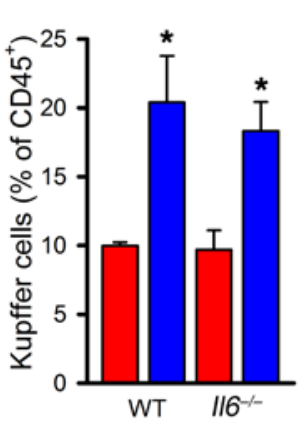

$\mathbf{F}$

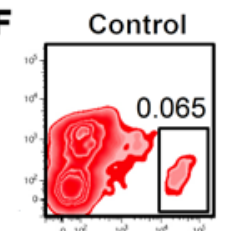

产
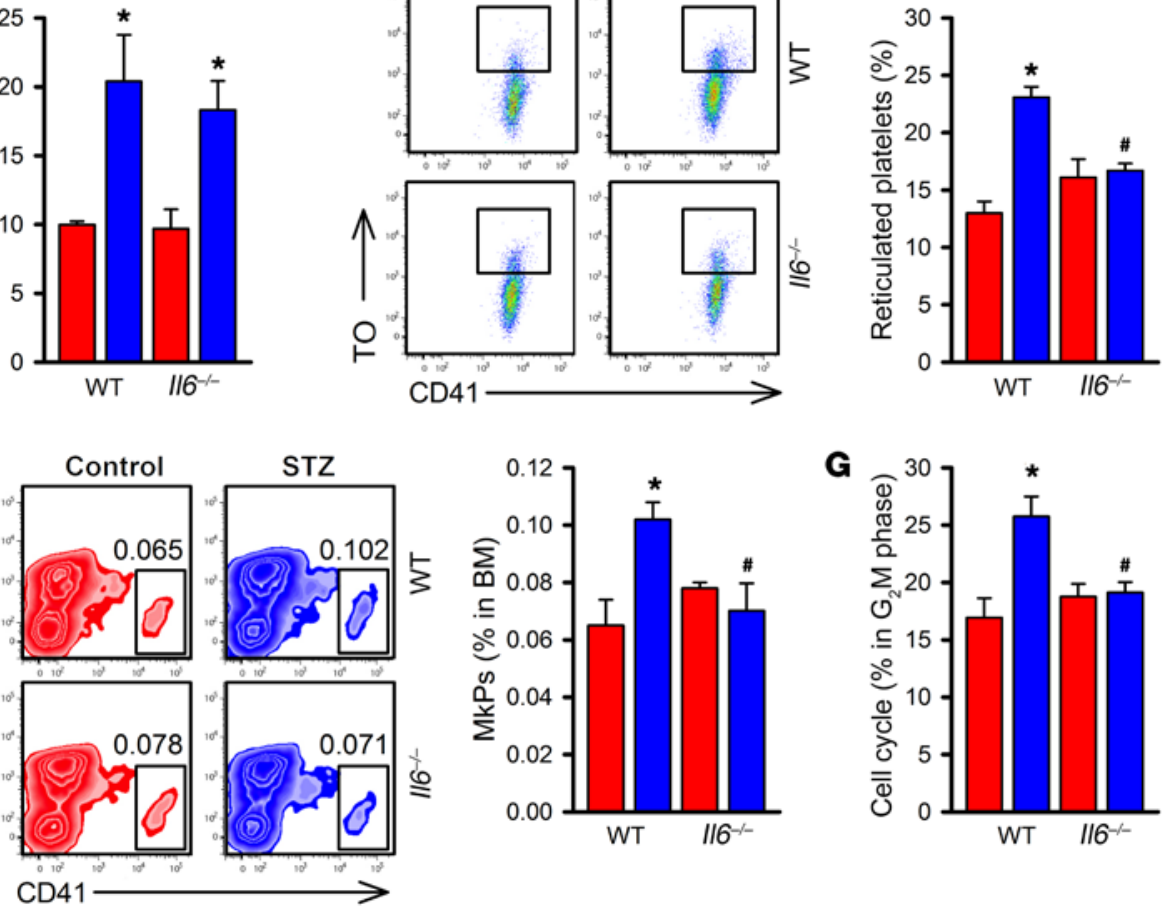

G

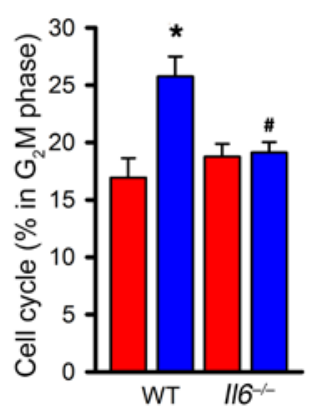

Figure 4. IL-6 drives reticulated thrombocytosis in diabetes. (A) Experimental overview: C57BL/6 WT recipient mice were transplanted with BM from WT or $116^{-1-}$ donor mice, and after 5 weeks a subset of mice were rendered diabetic with STZ. After 4 weeks the mice were analyzed. (B) Blood glucose levels. (C) Percentage of Kupffer cells in the liver was quantified by flow cytometry. (D and E) Percentage (D) and numbers (E) of circulating reticulated platelets. TO, thiazole orange. $(\mathbf{F}$ and $\mathbf{G})$ Abundance $(\mathbf{F})$ and proliferation $(\mathbf{G})$ of MkPs in the BM were quantified by flow cytometry. $n=5 \mathrm{WT}$ groups, $n=6 / 16^{-/-}$controls, and $n=10$ $116^{-/-}$STZ. ${ }^{*} P<0.05$ for disease effect, ${ }^{\#} P<0.05$ for genotype effect. All data represent the mean \pm SEM. $P$ values were obtained using a 2 -way ANOVA.

diabetic mice that received $I 6^{-1-} \mathrm{BM}$, similar to that observed in nondiabetic mice (Figure 4, D and E). This paralleled a reduction in the abundance and proliferation of BM MkPs (Figure 4, F and G). Together, these data confirm that IL-6 is a key mediator in diabetes-associated thrombocytosis.

RAGE mediates diabetes-associated thrombocytosis. Next, we wanted to identify the receptor that mediates IL-6 expression in Kupffer cells. We found that the expression of receptor for advanced glycation end products (RAGE) was significantly enhanced on the Kupffer cells from diabetic mice (Figure 5A), consistent with the increased inflammatory tone in the liver of these mice. To further investigate the role of RAGE, we rendered WT and Rage $^{-/-}$mice diabetic with STZ. Since our Rage ${ }^{-/}$mice express green fluorescent protein (GFP), we were unable to determine the abundance of reticulated platelets using thiazole orange. However, consistent with our hypothesis, there were fewer circulating platelets in the diabetic Rage-/ mice (Figure 5B). This was associated with fewer MEPs and MkPs within the BM (Figure 5C). These data highlight an important role for RAGE in diabetes-associated thrombocytosis. To further delineate the role of RAGE in diabe- tes-associated thrombocytosis, we performed BMT with WT mice reconstituted with BM from either Rage $e^{-/}$or WT mice. Further, we followed the same protocol and rationale as in the $\mathrm{Il6}^{--}$BMT studies; the mice were rendered diabetic after a 5 -week reconstitution period (Figure 5, D and E). After 4 weeks of diabetes, we found that Rage ${ }^{-/}$BMT diabetic mice had fewer platelets (Figure 5F), which was associated with fewer MkPs in the BM (Figure 5G). Furthermore, Rage $^{-1-}$ BMT mice were protected from diabetes-associated liver inflammation as characterized by fewer Kupffer cells (Figure 5H), which expressed less IL-6 (Figure 5I). Consistent with the reduced IL- 6 , the levels of TPO were significantly reduced in the plasma of the Rage-/- BMT mice (Figure 5J). To rule out the possibility that RAGE signaling induces a direct proliferative effect within the MEPs, we performed a competitive BMT (cBMT) study. Equal proportions of BM from WT CD45.1 and WT CD45.2 mice, or WT CD45.1 and Rage - $^{--}$CD 45.2, were mixed and transplanted into CD45.2 WT recipients, which were then made diabetic with STZ (Supplemental Figure 2A). Consistent with a non-cell-autonomous model of RAGE-induced proliferation (i.e., in the Kupffer cell), we observed that the abundance of both platelets and BM 
A

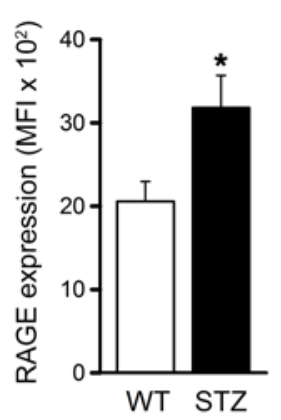

B

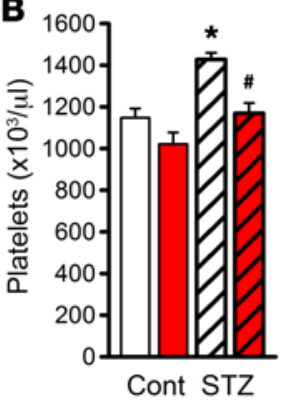

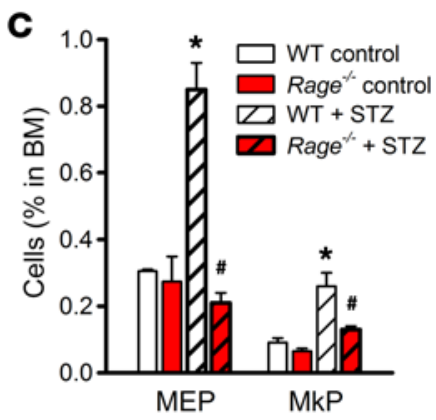

D Donors:

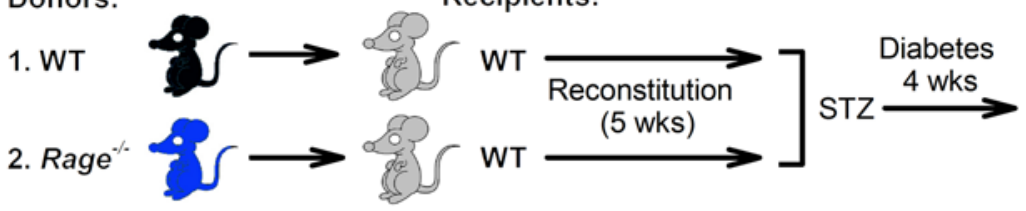

Figure 5. Deletion of Rage protects from thrombocytosis in diabetic mice. (A) RAGE expression on the surface of Kupffer cells from WT and age-matched 4-week-diabetic mice was quantified by flow cytometry. $n=6$ per group. ${ }^{*} P<0.05$. (B and C) WT and $\mathrm{Rage}^{-/-}$mice were made diabetic with $\mathrm{STZ}$, and 4 weeks later platelets (B) and BM MEPs and MkPs (C) were quantified. ${ }^{*} P<0.05$ vs. WT. ${ }^{\#} P<0.05$ vs WT+STZ. (D) Experimental overview: WT recipient mice were transplanted with BM from WT or $\mathrm{Rage}^{-/}$ mice and after a 5-week reconstitution period were rendered diabetic with STZ. After 4 weeks the mice were analyzed. (E) Blood glucose levels. (F) Circulating platelets. (G-I) Abundance of MkPs in the BM (G) and Kupffer cells in the liver (H) and Kupffer cell IL-6 expression (I) were quantified by flow cytometry. (J) Plasma TPO was quantified by ELISA. $n=7$ control, and $n=11$ diabetic. ${ }^{*} P<0.05$ vs. WT. All data represent the mean \pm SEM. $P$ values were obtained using a $t$ test, except in B and C, where 2-way ANOVA was used per cell type.
E

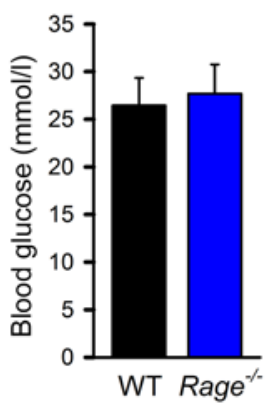

H

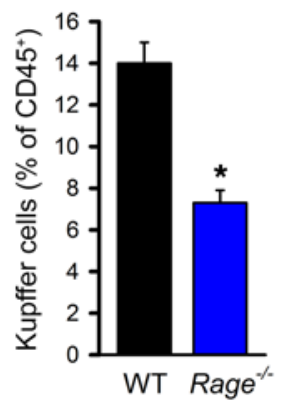

$\mathbf{F}$

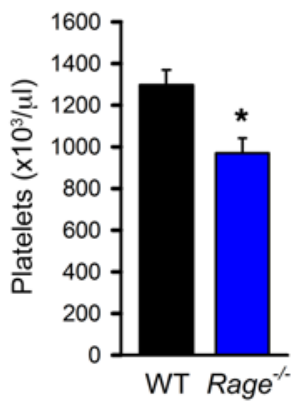

I

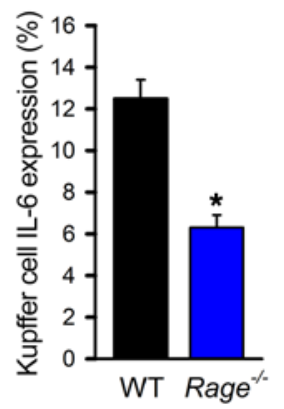

G

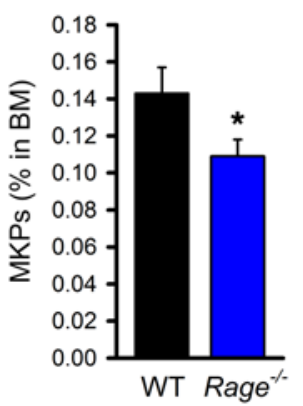

J

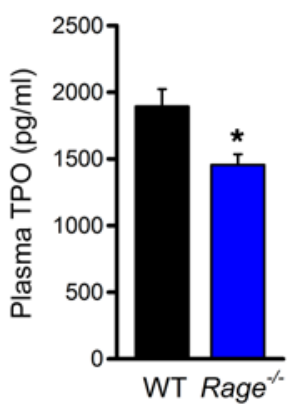

cium-binding proteins A8/A9 (S100A8/A9), which when released bind to RAGE on CMPs in the BM to promote leukocytosis (6). Since we identified RAGE on Kupffer cells, we sought to examine whether S100A8/A9 could induce IL-6 expression in macrophages. We cultured BM-derived macrophages (BMDMs, resembling Kupffer cells) under high-glucose conditions and stimulated them with recombinant S100A8/A9. We observed a significant increase in 16 mRNA expression in BMDMs treated with S100A8/A9 (Figure 6A). The data from cBMT (Supplemental Figure 2) studies revealed that RAGE was not directly involved in a proliferative response in the MkPs. To confirm this, we performed an in vitro MkP proliferation assay and found no direct proliferative role for S100A8/A9 on MkPs (Figure 6B). To formally test the role of S100A8/ A9 in diabetes-associated thrombocytosis, we compared diabetic WT and S100a9 ${ }^{-/-}$mice (also deficient in S100A8 protein) with WT controls (Figure 6C; glucose values). Interestingly, dele-

MEPs was reduced in the mice that received Rage ${ }^{-/-} \mathrm{CD} 45.2 \mathrm{BM}$, while the ratio of CD45.1- to CD45.2-derived cells was not significantly different between the WT CD 45.2 and Rage ${ }^{-1-}$ CD 45.2 transplanted chimeras (1.03 vs. 1.07, respectively; Supplemental Figure 2B). This indicates that the reduction in circulating platelets and BM MEPs observed in Rage ${ }^{-/}$BMT mice was due to a cell-extrinsic mechanism, specifically from RAGE expression in Kupffer cells. Further, there was no surface expression of RAGE detected on MEPs/MkPs from WT or diabetic mice. We have previously found that RAGE is expressed only on CMPs within the hematopoietic progenitor population (6). Overall, these data reveal an important role for RAGE in promoting thrombocytosis in diabetes.

Neutrophil-derived S100A8/A9 promotes thrombocytosis in diabetes. We next sought to identify the RAGE ligands that activate IL-6 signaling in the Kupffer cells. We have previously reported that hyperglycemia enhances neutrophil production of S100 cal- tion of S100a9 had no effect on the abundance of Kupffer cells (Figure 6D). However, the Kupffer cells from S100a9-/- mice failed to upregulate IL- 6 production in the setting of diabetes (Figure $6 \mathrm{E}$ ), and this was accompanied by a protection from diabetes-associated thrombopoiesis (Figure 6, F and G). Myeloid cells largely produce S100A8/A9; thus, we performed a BMT study using $5100 a 9^{-/} \mathrm{BM}$ to confirm that hematopoietic cells were providing the source of S100A8/A9 (Figure 6H). Similar to the global S100a9-/ mice, hematopoietic deletion of S100A9 also protected mice from diabetes-induced thrombocytosis. Indeed the S100 a $9^{-/-}$ BMT diabetic mice had reduced Kupffer cell IL- 6 expression and fewer reticulated platelets and BM MkPs, which were also proliferating less than those observed in the mice that received WT BM (Figure 6, I-L). Among the myeloid cells, neutrophils are the predominant source of the serum S100A8/A9 pool. To assess the specific contribution of neutrophils in promoting thrombopoiesis, 
A

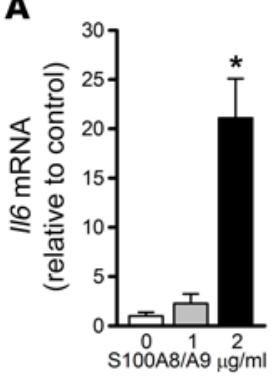

B

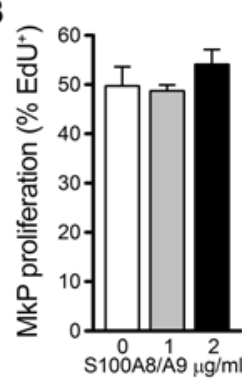

E
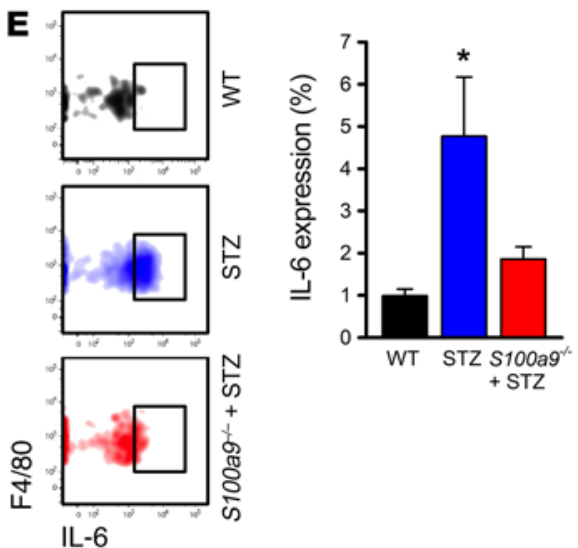

$\mathbf{F}$

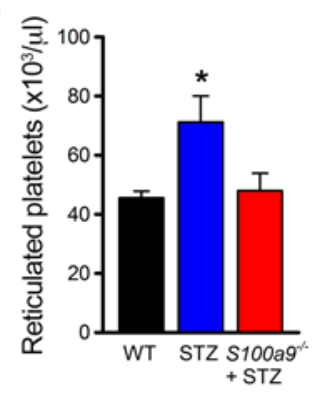

D
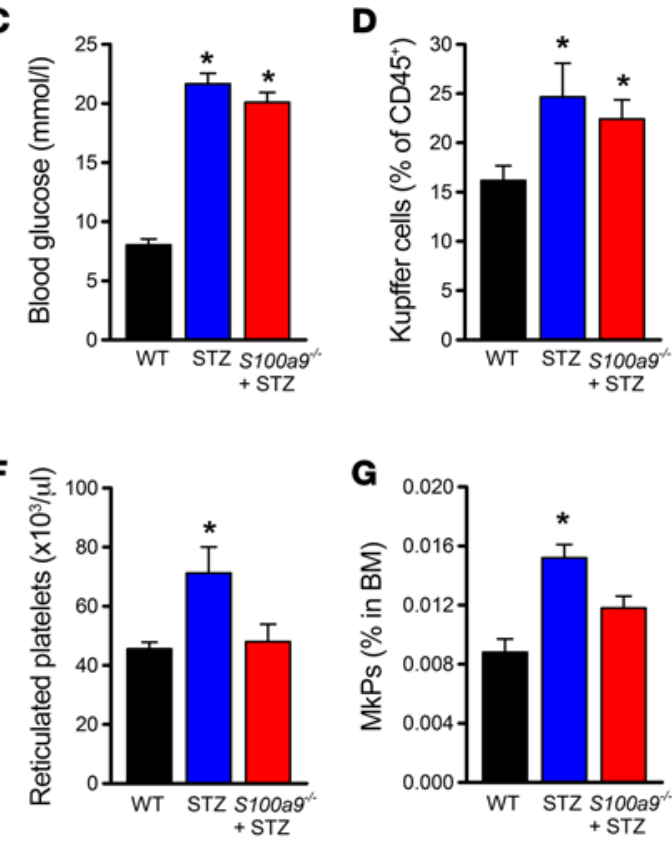

Figure 6. Neutrophil-derived S100A8/A9 promotes thrombocytosis in diabetic mice. (A) $/ / 6$ mRNA expression was quantified in BMDMs cultured in high glucose ( $25 \mathrm{mM}$ ) and incubated with S100A8/A9 for 6 hours. $n=4$. ${ }^{*} P<0.05$ vs. 0 . (B) $\mathrm{BM}$ progenitor cells were cultured in high glucose with S100A8/A9 for 16 hours. MkP proliferation was quantified via EdU incorporation. $n=3$ per group. (C-C) WT and S100a9-/- mice were made diabetic with STZ and analyzed after 4 weeks of diabetes. (c) Blood glucose levels. Livers were digested, and the abundance of Kupffer cells (D) and IL-6 expression (E) were quantified by flow cytometry. Circulating reticulated platelets (F) and BM MkPs (G) were measured by flow cytometry. $n=5 \mathrm{WT}, n=9$ STZ, and $n=10$ S100a9 $/-$ STZ. ${ }^{*} P$ $<0.05$ vs. WT (C and D) and vs. all groups (E-C). (H) Experimental overview: BM from WT or S100a $9^{-/-}$mice was transplanted into WT recipient mice, and after 5 weeks a subgroup of mice were rendered diabetic with STZ. (I-L) Kupffer cell IL-6 expression $(\mathrm{I})$, reticulated platelets (J), BM MkP abundance (K), and MkP proliferation (L) were quantified by flow cytometry. $n=5$ WT control, $n=5$ WT STZ, $n=45100 a 9^{-/-}$control, $n$ $=65100 a 9^{-1-}$ STZ. ${ }^{*} P<0.05$ for disease effect, ${ }^{\#} P<0.05$ for genotype effect. (M-Q) Neutrophil depletion studies: Neutrophils were depleted in WT and diabetic (STZ) mice after the onset of diabetes for 4 weeks by i.p. injection of the neutrophil-specific anti-Ly6-G antibody (clone 1A8 $1 \mathrm{mg} /$ injection, every 3 days), and these mice were compared with isotype control-treated mice. (M) Neutrophil levels were assessed by flow cytometry. (N) Plasma S100A8/ A9 levels. (0) Platelet levels were quantified by complete blood count. ( $\mathbf{P}$ and $\mathbf{Q})$ Abundance $(\mathbf{P})$ and proliferation (Q) of BM MEPs were quantified by flow cytometry. $n=5$ per group. ${ }^{*} P<0.05$ for disease effect, ${ }^{\#} P<0.05$ for treatment effect. All data represent the mean \pm SEM. $P$ values were obtained using 1-way ANOVA (A-C) or 2-way ANOVA (I-Q).

we performed a neutrophil depletion study using an anti-Ly6-G antibody (Figure 6, M-Q). Similar to our previous findings, diabetic mice had significantly higher levels of plasma S100A8/A9 that was neutrophil-derived (Figure 6N) (6). Consistent with our hypothesis, neutrophil-depleted diabetic mice had significantly lower levels of circulating platelets and fewer BM MEPs that were proliferating at a lower rate compared with isotype control-injected mice (Figure 6, O-Q). Together, these data indicate an important role for neutrophil-derived S100A8/A9 in promoting platelet production in diabetes. 


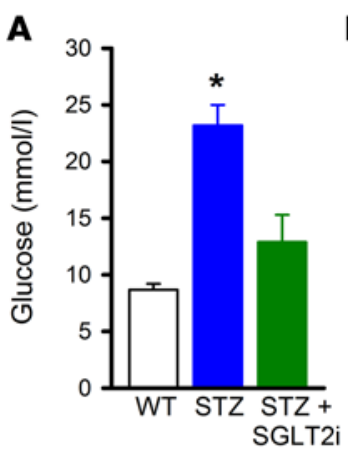

E

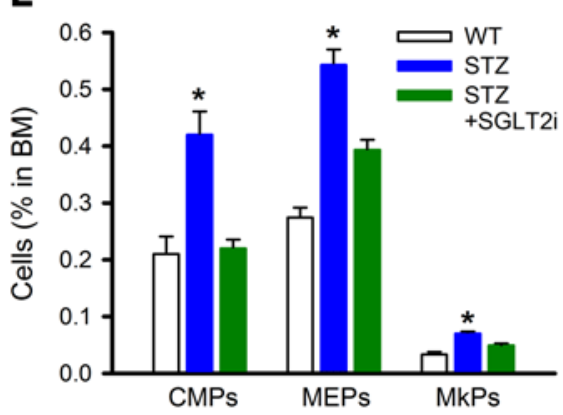

H

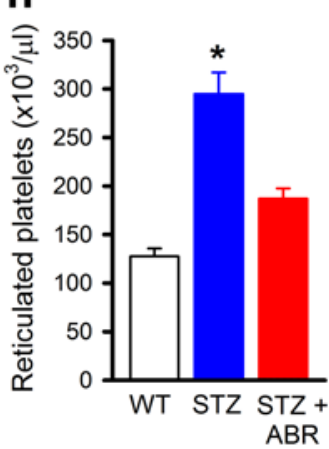

B

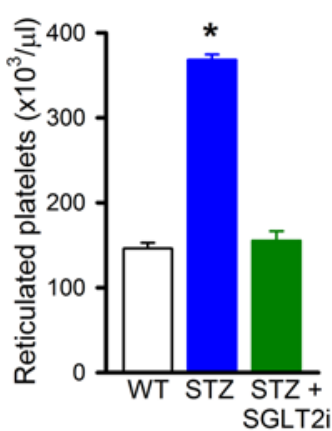

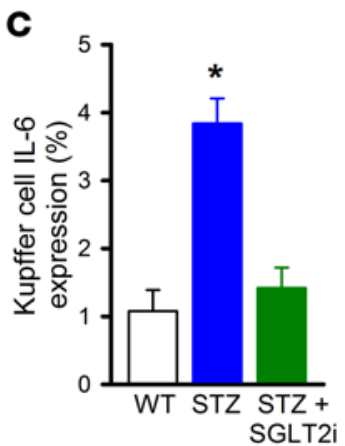

$\mathbf{F}$

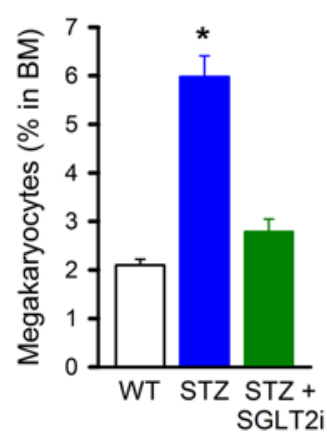

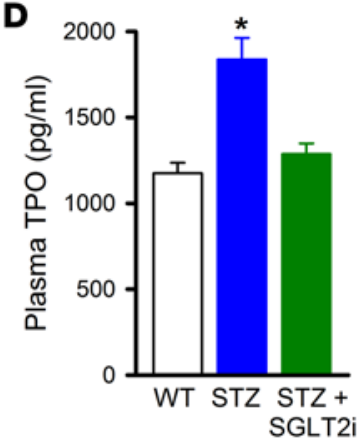

G

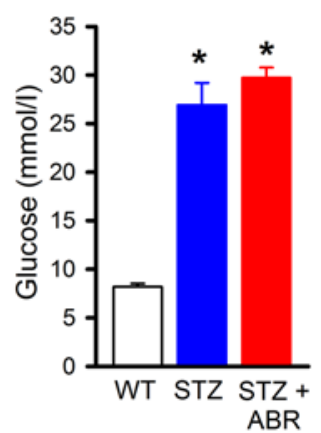

$\mathbf{K}$

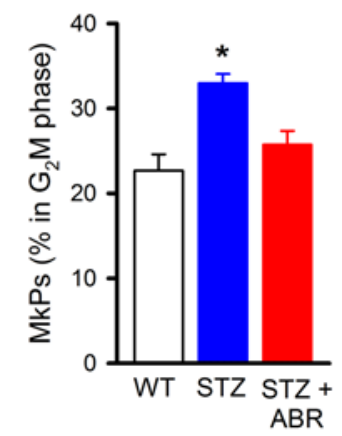

Figure 7. Interventions to inhibit thrombocytosis in diabetic mice. (A-F) SGLT2i studies: C57BL/6 mice were made diabetic with STZ, and blood glucose was lowered with the SGLT2i dapagliflozin (25 mg/kg/d) in the drinking water for 4 weeks. (A) Blood glucose levels. (B and C) Circulating reticulated platelets (B) and Kupffer cell IL-6 expression (C) were measured by flow cytometry. (D) Plasma TPO levels were quantified by ELISA. (E and F) BM progenitor cells (E) and megakaryocytes (F) were quantified by flow cytometry. $n=6$ control and STZ+SGLT2i and $n=4$ STZ. ${ }^{*} P<0.05$ vs. all groups. (G-K) ABR-215757 studies: C57BL/6 mice were made diabetic with STZ and treated with ABR-215757 (10 mg/kg/d) or vehicle in the drinking water for 4 weeks. (C) Blood glucose levels. Circulating reticulated platelets (H) and Kupffer cell IL-6 expression (I) were measured by flow cytometry. The abundance (J) and proliferation (K) of MkPs in the BM were quantified by flow cytometry. $n=6$ control and $n=9$ STZ and STZ+ABR. ${ }^{*} P<0.05$ vs. WT (G) and vs. all groups (H-K). All data are mean \pm SEM. $P$ values were obtained using 1-way ANOVA.

Reticulated thrombocytosis is also observed in models of obesity/ insulin resistance/pre-type 2 diabetes. To explore whether reticulated thrombocytosis was also observed in other common metabolic disorders, namely obesity, insulin resistance, and type 2 diabetes (T2D), we quantified platelets in 24-week-old male WT, ob/ob, and diet-induced obese (DIO) mice (16 weeks of high-fat feeding). The $o b / o b$ and DIO mice demonstrated reticulated thrombocytosis, consistent with observations in humans that reticulated thrombopoiesis is not specific to insulin deficiency (Supplemental Figure 3, $\mathrm{A}-\mathrm{C})$. We further explored the livers of the DIO mice and extended our Kupffer cell analysis to include a newly discovered marker, C-type lectin domain family 4 member F (CLEC4F) (Supplemen- tal Figure 3D), which was unfortunately not reported before the bulk of the data were collected in this study. However, consistent with our mechanism, we observed a significant increase in these CLEC4F ${ }^{+}$Kupffer cells and IL-6 expression (Supplemental Figure 3, D and E). Importantly, we also confirmed that the CLEC4F ${ }^{+}$ Kupffer cells were increased in the STZ model (Supplemental Figure 3, F and $\mathrm{G}$ ), suggesting that the analysis of our models prior to the discovery of this marker was still valid.

Reducing serum glucose improves reticulated thrombocytosis in diabetes. To test whether hyperglycemia is responsible for reticulated thrombocytosis, we treated diabetic mice with the sodium-glucose cotransporter 2 inhibitor (SGLT2i), dapagliflozin, 
A

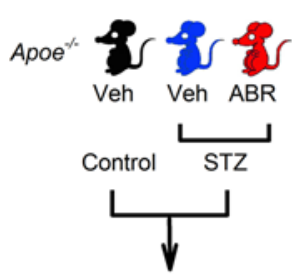

Chow diet 12 weeks

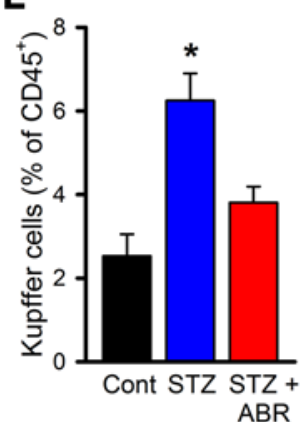

I

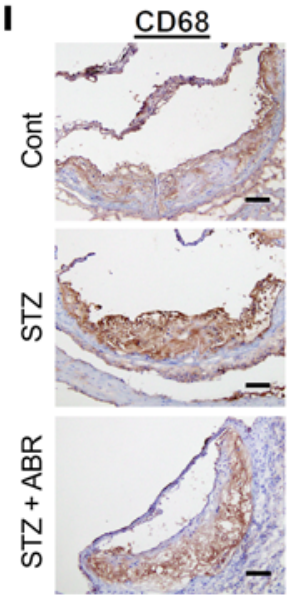

K

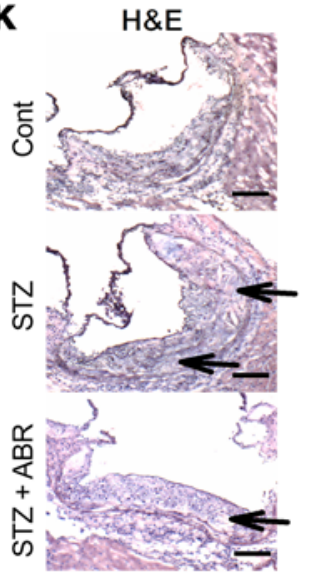

B

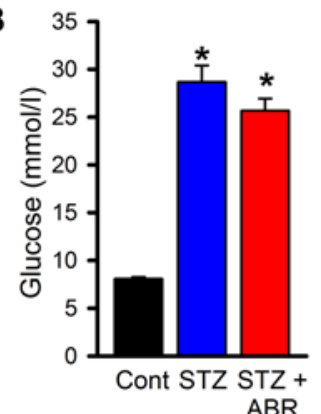

$\mathbf{F}$

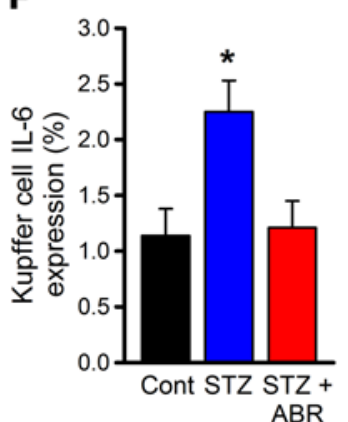

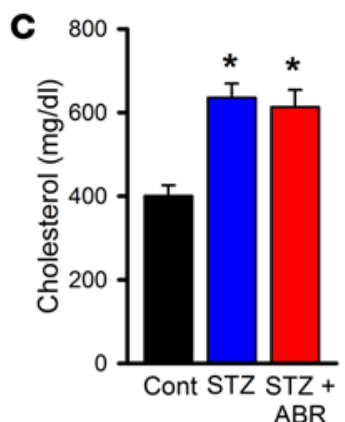

G

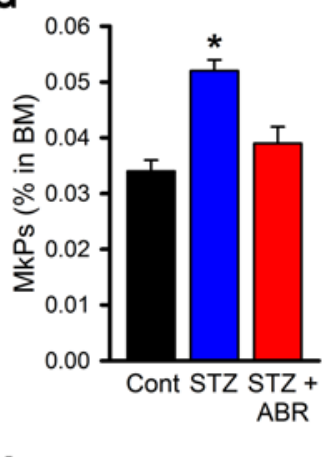

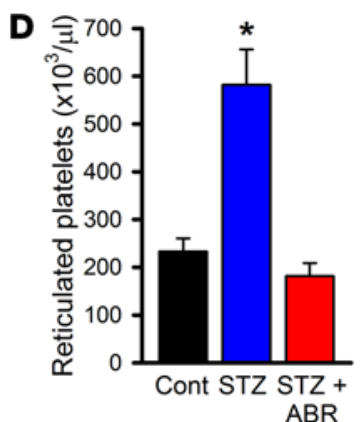

H

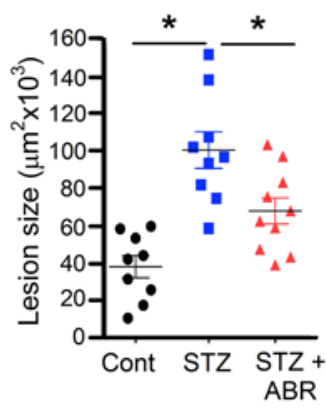

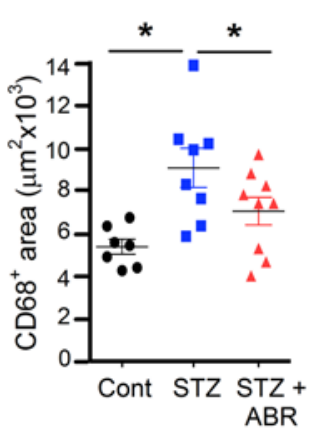

J
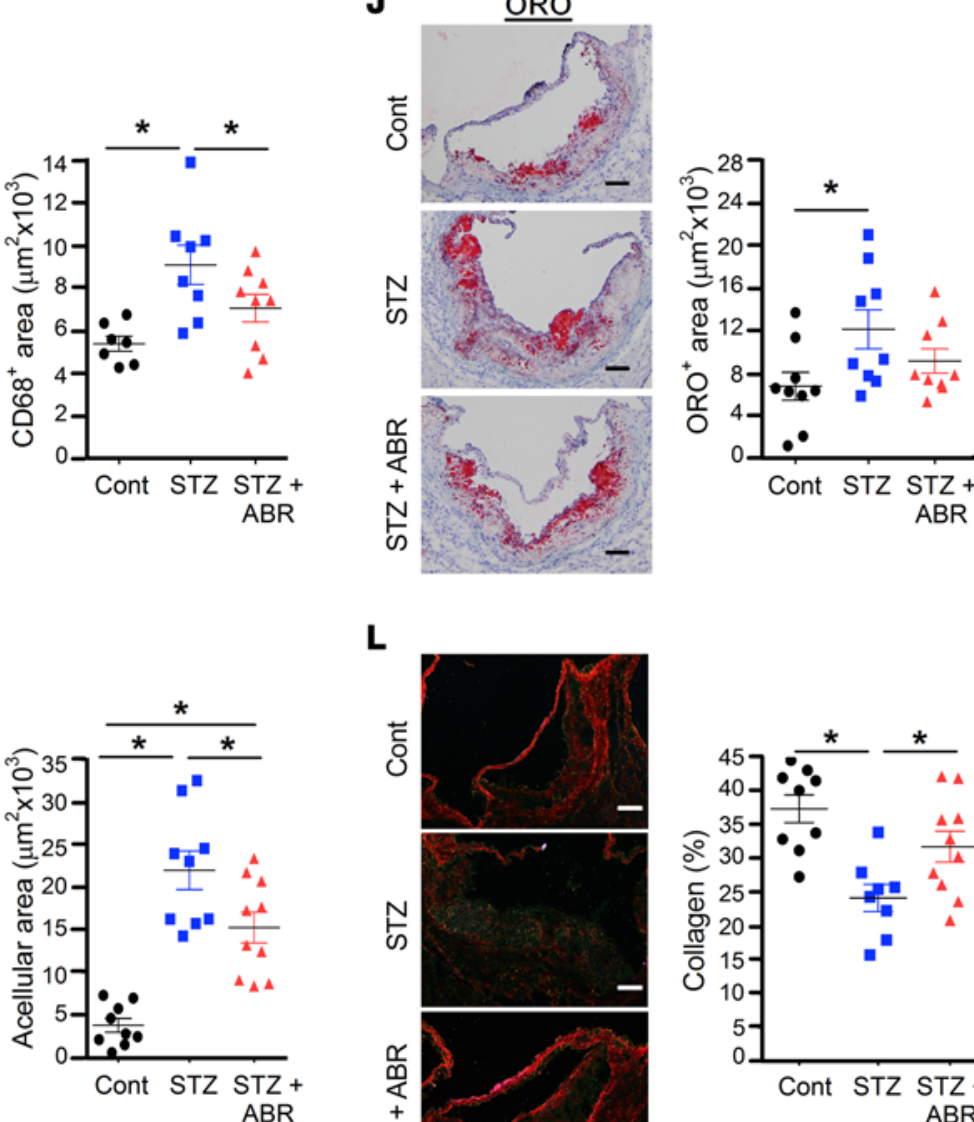

$\mathbf{L}$

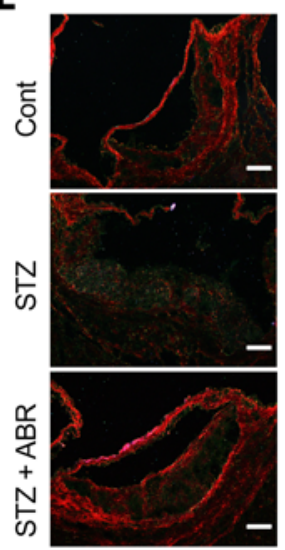

Figure 8. Blocking S100A8/ A9 with ABR-215757

decreases atherogenesis in diabetic mice. (A) Experimental overview: 8-week-old $A p o e^{-/-}$mice were divided into 3 groups, vehicle (Cont), vehicle diabetic (STZ), and ABR-215757 diabetic (STZ+ABR; $10 \mathrm{mg} / \mathrm{kg} / \mathrm{d}$ in drinking water), and fed a chow diet for 12 weeks. (B and C) Blood glucose levels (B) and plasma cholesterol levels (C). (D-G) Circulating reticulated platelets (D), Kupffer cells (E), Kupffer cell IL-6 expression (F), and BM $\operatorname{MkPs}(\mathbf{C})$ were measured by flow cytometry. (H) Quantification of mean lesion size. (I and J) Macrophage abundance was assessed by area of CD68+ regions (I) and lipid content by Oil Red $\mathrm{O}^{+}$ area (J). (K) Acellular regions were identified in H\&E stain lesions (see arrows). (L) Percentage collagen content in the lesions was assessed by Picrosirius red staining and visualized under polarizing light. $n=9$ control and STZ and $n=10 \mathrm{STZ}+\mathrm{ABR} .{ }^{*} P<$ 0.05 vs. WT (B and C), vs. all groups (D-G), or as indicated by lines $(\mathbf{H}-\mathbf{L})$. All data represent the mean \pm SEM. $P$ values were obtained using a 1-way ANOVA. Scale bars: $100 \mu \mathrm{m}$. which lowers blood glucose in an insulin-independent manner. The SGLT2i reduced blood glucose levels of the diabetic mice to levels comparable to those of the WT control mice (Figure 7A). The reduction in blood glucose by SGLT2i treatment normalized reticulated platelet levels (Figure $7 \mathrm{~B}$ ). We also observed that the Kupffer cells were producing less IL-6 (Figure 7C), which was associated with a significant reduction in circulating TPO (Figure 7D). Consistent with the reduction in circulating TPO, glucose 
Table 1. Reticulated platelets and leukocyte interactions in people with type 2 diabetes

$\begin{array}{lccc} & \text { Control }(\boldsymbol{n}=14) & \text { Diabetes }(\boldsymbol{n}=10) & P \text { value } \\ \text { Platelets }\left(\times 10^{9} \text { per liter }\right) & 219 \pm 49.3 & 259 \pm 69.6 & 0.117 \\ \begin{array}{l}\text { Reticulated platelets }(\%) \\ \text { Reticulated platelets }\end{array} & 6.6 \pm 3.4 & 11.1 \pm 4.5 & 0.0102 \\ \begin{array}{l}\left(\times 10^{9} \text { per liter }\right) \\ \text { CD14 }\end{array} & 14.4 \pm 8.6 & 32.0 \pm 16.7 & 0.0047 \\ \text { aggregates }(\%) & 11.2 \pm 4.9 & 23.2 \pm 12 & 0.0032\end{array}$

Data are shown as the mean \pm SD. $P$ values were obtained using a $t$ test.

lowering with SGLT2i reduced the percentage of BM progenitor cells as well as megakaryocytes (Figure 7, E and F). Together, these data reveal that diabetes-associated reticulated thrombocytosis can be ameliorated with improved glucose control. These results are consistent with our previous findings that MPV is increased in people with poor diabetes control (40).

ABR-215757 reduces diabetes-associated thrombocytosis. We have identified a prominent role for S100A8/A9 in stimulating IL-6 production in Kupffer cells to evoke thrombopoiesis (Figure 6). To determine whether S100A8/A9 is an effective target to prevent reticulated thrombocytosis, we tested a small-molecule inhibitor, ABR-215757 (paquinimod), that blocks the binding of S100A9 to TLR4 and RAGE (41). ABR-215757 (10 mg/kg/d) was administered to STZ diabetic mice in the drinking water for 4 weeks. Administration of ABR-215757 had no impact on blood glucose levels compared with those in nontreated diabetic mice (Figure 7G). Nevertheless, blocking S100A8/A9 with ABR-215757 led to a significant reduction in reticulated platelets (Figure $7 \mathrm{H}$ ). Consistent with our proposed model, ABR-215757 treatment reduced Kupffer cell IL-6 expression, which was accompanied by fewer $\mathrm{BM}$ MkPs that were proliferating at reduced rates (Figure 7, I-K). These data confirm that S100A8/A9 could be a potential target to reduce diabetes-associated thrombocytosis by inhibiting hepatic IL-6-mediated inflammation.
ABR-215757 inhibits diabetes-associated atherosclerosis. To determine whether blocking S100A8/A9 signaling would indeed result in a decrease in atherogenesis, we performed a study in atherosclerotic prone $\mathrm{Apoe}^{-/-}$mice. Eight-week-old mice were fed a chow diet and randomized into three groups: control (vehicle), diabetic (STZ; vehicle), and diabetic plus ABR-215757 (10 $\mathrm{mg} / \mathrm{kg} / \mathrm{d}$ in drinking water) (Figure $8 \mathrm{~A}$ ). As expected, the diabetic mice had higher blood glucose levels and slightly elevated plasma cholesterol compared with the nondiabetic controls with these biochemical parameters not affected by ABR-215757 (Figure $8, \mathrm{~B}$ and $\mathrm{C}$ ). Consistent with the study above, we saw a dramatic decrease in reticulated platelets (Figure 8D), which was accompanied by fewer Kupffer cells and less IL- 6 expression, along with fewer BM MkPs (Figure 8, E-G). We next examined the atherosclerotic lesion size and complexity. In line with our hypothesis, atherosclerotic lesion size, which was dramatically increased in diabetic mice, was significantly reduced when the diabetic mice were treated with ABR-215757 (Figure 8H and Supplemental Figure 4). Importantly, ABR-215757 also decreased the abundance of plaque macrophages and tended to decrease lipid content (Figure 8 , I and J). We also observed larger acellular regions in the diabetic mice, which was reduced in the ABR-215757 treated mice. While the acellular regions in the ABR-215757 mice were still significantly larger than seen in the control mice, this appeared to be due to positive remodeling, since we detected an increase in collagen deposition, potentially stabilizing the lesion (Figure 8, K and L).

Subjects with $T 2 D$ have reticulated thrombocytosis and increased S100A8/A9 levels. In our preclinical models we have discovered an important role for S100A8/A9 in driving reticulated thrombopoiesis. Furthermore, we have previously reported that S100A8/ A9 levels are increased in a cohort of individuals with type 1 diabetes (T1D) with complications (6). In this study we explored whether there was an association between plasma S100A8/A9 and the abundance of reticulated platelets in people with T2D when compared with age-matched healthy subjects. As expected, people with T2D had moderate elevation in total platelet levels and a significant increase in the abundance of reticulated platelets (Table 1), consistent with our preclinical data (Figure 1, C
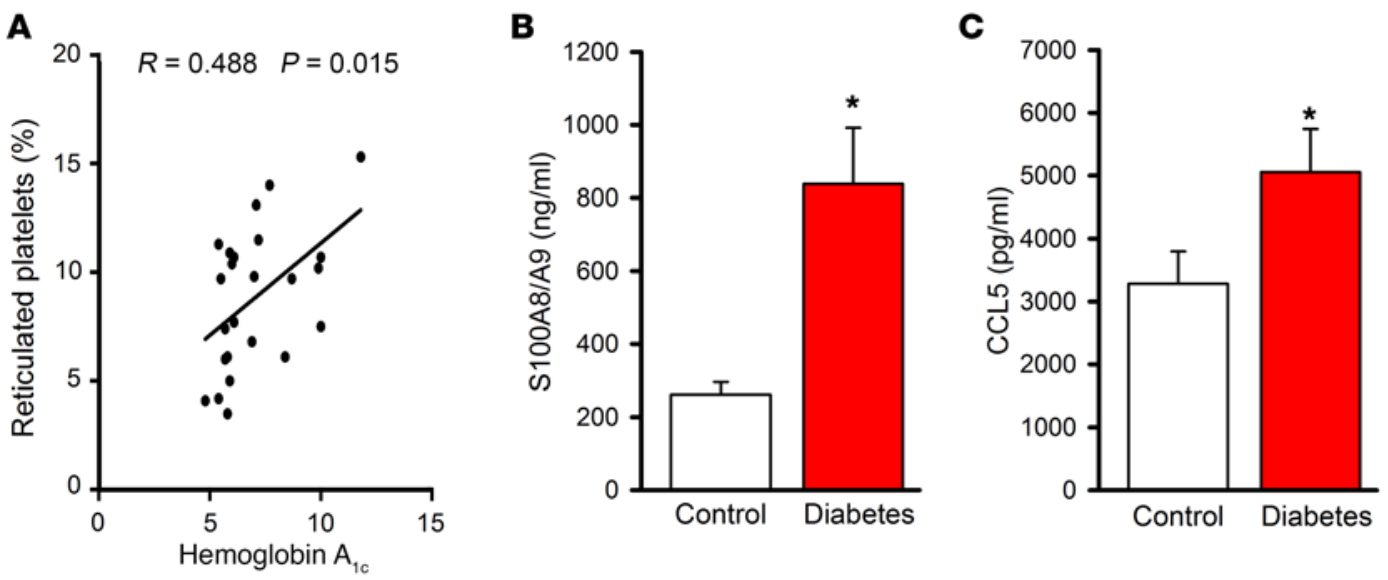

Figure 9. Reticulated thrombocytosis in people with type $\mathbf{2}$ diabetes. (A) Regression analysis of reticulated platelets versus hemoglobin $A_{1 c} ; n=24$. (B and C) Plasma S100A8/A9 (B) and CCL5 (C) levels were quantified by ELISA. $n=15$ control and $n=11$ diabetes. ${ }^{*} P<0.05$ vs. control. All data are mean \pm SEM. $P$ values were obtained using a $t$ test or linear regression (A). 


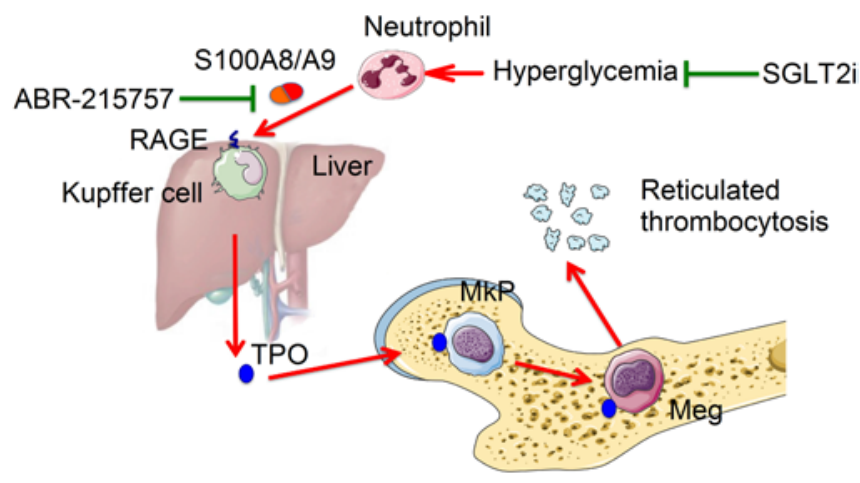

Figure 10. Proposed mechanism. Schematic overview of the proposed mechanism of diabetes-associated thrombocytosis (red arrows) and interventions (green blunted arrows). Meg, megakaryocyte.

and D, and Supplemental Figure 3), signifying enhanced platelet production in these individuals. We also observed more CD14 monocyte-platelet aggregates, consistent with our animal studies, suggesting that these platelets were circulating in an activated state (Table 1 and ref. 42). Importantly, we found a correlation between reticulated platelets and hemoglobin $A_{1 c}$ levels (Figure $9 \mathrm{~A})$, consistent with the hypothesis that poor glycemic control is associated with enhanced thrombopoiesis in people with diabetes. Next we measured plasma S100A8/A9 levels and found significantly increased levels in this cohort (Figure 9B). Again, consistent with our preclinical findings, people with diabetes also had significantly higher plasma levels of the potent chemokine CCL5 (Figure 9C). Together, these findings in our T2D patient cohort reflect those seen in our preclinical models and are consistent with the view that the same mechanisms of enhanced platelet production are conserved in humans.

\section{Discussion}

Diabetes is an insidious disease that accelerates a number of vascular complications, with the major outcome being increased mortality due to atherosclerotic CVD. Platelets are well known for their role in CVD, particularly in thrombus formation, but also in driving the initiation and progression of atherosclerotic lesions $(8,10)$. In general, antiplatelet therapies significantly reduce the risk of CVD, but the effect of these drugs is somewhat attenuated in people with diabetes, perhaps because they have enhanced production of new platelets (16-18). Further, reticulated thrombocytosis could be an important factor driving accelerated atherosclerosis in diabetes. In this study we have identified that diabetes-induced liver inflammation promotes TPO production, which in turn stimulates thrombopoiesis within the BM. One of the initiating ligands in this pathway was identified as S100A8/A9, which is mainly derived from neutrophils in the setting of hyperglycemia. Excitingly, we discovered 2 tractable approaches, firstly by lowering blood glucose with an SGLT2i and secondly by preventing the bioactivity of S100A8/ A9 with the drug ABR-215757, could effectively normalize reticulated platelet levels in diabetic mice (Figures 7 and 10). Furthermore, administration of ABR-215757 mitigated the accelerated atherogenesis seen in diabetes.
Diabetes is a disease that perpetuates an inflammatory state (6) and, despite widespread cholesterol lowering in these subjects, is associated with a persistent increased risk of CVD (43). This residual risk of CVD in diabetes implies that there remains a major gap in our understanding of the underlying mechanisms responsible for increased CVD in diabetes. It is well established that people with diabetes have differences in their sensitivity and response to antiplatelet therapies. Increased platelet activation, particularly the release of platelet factor 4 from $\alpha$-granules, is well documented in people with diabetes $(44,45)$, along with increased number of platelets, particularly reticulated platelets and MPV $(14,16-19,21,22)$. These published findings were confirmed in our cohort of patients with T2D (Table 1). It is likely that the increase in reticulated platelets plays a causative role in accelerating CVD, since a study of over 200,000 individuals revealed that MPV was predictive of mortality due to ischemic heart disease (46). Moreover, the increase in reticulated platelets is suggested to be one of the main reasons for the decreased efficacy of certain antiplatelet drugs, including aspirin and clopidogrel, as seen in diabetes $(13,14,18,47)$.

We have recently linked reticulated thrombocytosis to accelerated atherosclerosis in various genetically deficient (i.e., $A b c g 4^{-1-}$ and $\left.A b c b 6^{--}\right)$preclinical models $(10,48)$. The mechanism appears to relate to increased platelet-leukocyte aggregates, leukocyte activation, and plasma levels of the potent leukocyte chemokine CCL5. Further, it has been shown that deletion of CCL5 results in smaller atherosclerotic lesions (9). Interestingly, people with myeloproliferative neoplasms, including those biased towards enhanced platelet production, such as essential thrombocytosis and polycythemia vera, often die of atherothrombotic disease (49). Our data are reminiscent of the platelet dynamics seen in people with these conditions, where activating JAK2 mutations (enhanced JAK/ STAT signaling) cause thrombocytosis along with enhanced clearance by monocytes and neutrophils (50). The enhanced level of platelets in these aforementioned studies was due to cell-intrinsic mutations in MkPs and other BM progenitors resulting in uncontrolled proliferation. However, in the setting of diabetes we did not find this phenomenon, with no intrinsic differences in the MkPs. This suggests that external cues are responsible for the enhanced proliferation of these progenitors in diabetes. Accordingly, we detected increased plasma levels of the platelet-producing cytokine TPO, as has previously been reported in people with diabetes (51). This finding pointed to a role for the liver, the major source of TPO. Generally, an increase in platelet activation or preactivated platelets would result in enhanced clearance.

Previous studies have shown that in response to inflammation the liver can dramatically upregulate TPO, which then instructs the $\mathrm{BM}$ progenitors to increase the production of myeloid cells, particularly platelets (29). IL-6 is the major inflammatory cytokine that stimulates hepatic TPO production (31), which has been shown in genetically deficient mice and by administration of IL-6 to patients with advanced malignancies (30). Moreover, paraneoplastic thrombocytosis in people with epithelial ovarian cancer is also driven by IL-6 (tumor derived) and by induction of hepatic TPO (52). While systemic IL-6 levels could stimulate hepatocytes to upregulate $\mathrm{IL}-6$, there remains controversy as to the circulating levels of IL- 6 in the setting of diabetes. Thus, we hypothesized that 
local IL-6 production was more likely to induce TPO production. The liver is recognized as an important immune organ, and the hepatic specialized macrophages known as Kupffer cells appear to play a central role in recognizing invading pathogens (53), which in turn induce IL-6 expression (54). Indeed, we observed that not only were there more Kupffer cells in the livers of the diabetic mice, which were likely to have been recruited from the circulation (38), but these cells were also manufacturing greater amounts of IL-6. We confirmed the role of Kupffer cells through depletion studies using clodronate liposomes (37), which have been previously used to confirm the source of IL- 6 during listeria infections (54). Using a BMT approach, we also showed that engrafted $\mathrm{Il}^{-/-}$ Kupffer cells failed to respond to the hyperglycemic environment to induce reticulated thrombocytosis, confirming the importance of IL-6 in influencing platelet production.

Examining the pathways upstream of IL-6 led us to investigate RAGE, which we had previously shown to play an important role in diabetes-induced monocytosis by acting directly on CMPs in the BM (6). Competitive BMT studies ruled out a direct role for RAGE in promoting thrombocytosis at the progenitor cell level, confirming a role for peripheral signaling (i.e., hepatic). The liver is an important site of sterile inflammation, where pattern recognition receptors and damage-associated molecular patterns (DAMPs) drive inflammatory responses (55). We found that RAGE was significantly upregulated on Kupffer cells from diabetic animals. Deletion, both globally or specifically within the hematopoietic compartment, resulted in protection from diabetes-induced reticulated thrombocytosis. Importantly, we found that livers from Rage - $^{--}$BMT diabetic mice contained fewer Kupffer cells, which were also expressing less IL- 6 and had lower plasma TPO levels. Indeed, RAGE has not been implicated in contributing to platelet production. Nevertheless, previous studies have revealed that RAGE signaling on platelets can result in their activation, promoting a thrombotic phenotype and potentially encouraging leukocyte recruitment to the activated endothelium (56).

To further explore the clinical translational potential of these findings, human studies in T2D were performed. Indeed, we observed that platelet activation was increased in T2D subjects and contributed to enhanced monocyte-platelet aggregates. RAGE is a promiscuous receptor binding to a host of ligands, including AGEs, HMGB1, and S100A8/A9. Interestingly, these ligands can also signal via other pattern recognition receptors including TLR4 and CD36. The selectivity appears to be disease and tissue dependent, such that we have previously determined that S100A8/A9 is a potent RAGE ligand in the setting of hyperglycemia (6), but binds to TLR4 in obesity (57). In a series of elegant studies, deletion of S100A9 was shown to protect against ischemia, vasculitis, and atherosclerosis (58). S100A8/A9 has also been shown to regulate thrombosis during an acute myocardial infarction, but this effect appears to be mediated through platelet-expressed CD36 in the setting of normoglycemia (59). Importantly, S100A9 was found not to be involved in hemostasis, suggesting that targeting this pathway will not result in uncontrolled bleeding (59). Nevertheless, it appears that S100A8/A9 are key mediators of platelet activation, and furthermore we have now demonstrated that these DAMPs are also important regulators of enhanced platelet production specifically in diabetes.
S100A8 and S100A9 are largely produced by neutrophils, particularly in the setting of diabetes (6). Through genetic deletion of S100a9 by a BMT approach, we narrowed down the role for S100A8/A9 from myeloid cells. Indeed, using neutrophil depletion studies we confirmed that neutrophils were the dominant source of these DAMPs. Neutrophils are almost exclusively glycolytic and thus are sensitive to ambient glucose concentrations. We have previously shown that normalizing blood glucose levels with an SGLT2i prevents the accumulation of reactive oxygen species (6), classical by-products of activated and stressed neutrophils. Using this approach, we found that normalization of blood glucose in an insulin-independent manner with the SGLT2i prevents the activation of the thrombopoietic pathways that we had identified. Furthermore, we confirmed that poor glycemic control in people with T2D is associated with increased MPV $(40,60,61)$, a marker of more reticulated platelets. Our mouse models of obesity/insulin resistance/T2D revealed a similar mechanism at the level of the Kupffer cell, but the upstream initiating factors could be different.

While the overall management of diabetic patients has improved over the past few decades, maintaining glucose control in the optimal range still remains difficult. Epidemiological data have revealed an important contribution of postprandial hyperglycemic periods to increased cardiovascular risk (62-66). The efficacy of SGLT2i's not only on HbA1c levels, but more importantly glycemic variability remains to be determined. However, the recently published EMPA-REG study has shown that the SGLT2i empagliflozin significantly decreased the incidence of cardiovascular events, including cardiovascular death, in people with diabetes (67). The underlying explanation for decreased cardiovascular mortality with SGLT2 inhibition is not fully understood. Indeed, the key findings could be linked at least in part to actions on the atherothrombotic process, specifically thrombosis as a result of reduced reticulated platelets.

As an alternative approach independent of glucose control, we directly targeted the initiating ligands, S100A8/A9, with the small-molecule inhibitor ABR-215757. This inhibitor has been granted orphan medicinal product status in Europe and the US for the indication of systemic sclerosis. Importantly, ABR-215757 has been reported to have few side effects and encouraging pharmacokinetics in early-phase trials in people with systemic lupus erythematosus (68). Importantly, we now show that this molecule inhibited atherogenesis in a preclinical model of diabetes, providing a potential new therapeutic approach to reduce the cardiovascular burden in diabetes. However, we should note that the reduction in atherosclerosis induced by ABR-215757 could also be via additional mechanisms and requires further investigation.

In summary, our study reveals that reticulated thrombocytosis in diabetes is due to enhanced production in the BM. We identified that poor glycemic control stimulates neutrophil-derived S100A8/A9 to act on Kupffer cells to stimulate hepatic TPO in an IL-6-dependent manner (Figure 10). Through the use of the glucose-lowering agent dapagliflozin (SGLT2i) or by inhibiting the bioactivity of S100A8/A9 (ABR-215757), we could dampen platelet production. Therefore it is anticipated that a reduction in these reactive reticulated platelets should lead to increased efficacy of current antiplatelet therapies and in turn reduce the incidence and severity of cardiovascular events, which remains the major 
cause of morbidity and mortality in this population. By identifying S100A8/A9 as major glucose-responsive elements that play a significant role in myelopoiesis (6) and now thrombopoiesis, we propose S100A8/A9 as a promising target to manage cardiovascular complications in diabetes.

\section{Methods}

Mice and treatments. Male mice were about 8 weeks of age when entered into the studies. WT C57BL/6, Rage $^{-/}, \mathrm{Il6}^{-/}, \mathrm{S1OOA9}^{-/}$, and $o b / o b$ (The Jackson Laboratory) mice were bred and housed in the Alfred Medical Research Education Precinct Animal Centre in a pathogen-free facility under controlled conditions and exposed to a 12-hour/12-hour light/dark cycle and were fed a standard chow, or in the case of the DIO mice a $60 \% \mathrm{kcal}$ diet (Specialty Feeds). The totalbody $\mathrm{S} 10 \mathrm{Oa} 9^{-/}$experiments were conducted at New York University.

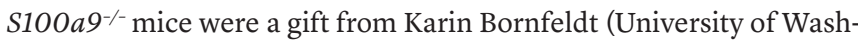
ington, Seattle, Washington, USA) originality created by Johannes Roth (University of Munster, Munster, Germany) (69).

Diabetes was induced by streptozotocin (STZ; $50 \mathrm{mg} / \mathrm{kg}$ i.p. injection for 5 days). The sodium-glucose cotransporter 2 inhibitor (SGLT2i) (dapagliflozin) was a gift from Bristol-Myers Squibb and was administered in the drinking water at a dose of $25 \mathrm{mg} / \mathrm{kg} / \mathrm{d}$ for 4 weeks.

Kupffer cell depletion. Liposomes containing either clodronate or PBS were injected via the tail vein $(250 \mu \mathrm{l} /$ mouse $)$ into a group of control (nondiabetic) and diabetic mice at day 1 and day 3 to deplete Kupffer cells.

ABR-215757 treatment. ABR-215757 was a gift from Active Biotech and was used to therapeutically inhibit the bioactivity of S100A8/A9. ABR-215757 was added to the drinking water to deliver an approximate dose of $10 \mathrm{mg} / \mathrm{kg} / \mathrm{d}$.

Reticulated platelets were measured as previously described (10). Undiluted EDTA-anticoagulated blood $(5 \mu \mathrm{l})$ within 30 minutes of collection was mixed with an anti-CD41-APC antibody (eBioscience) and thiazole orange (final concentration $1 \mu \mathrm{g} / \mathrm{ml}$ ) and incubated at room temperature in the dark for 20 minutes. Reticulated platelets were identified as CD41+ thiazole orange ${ }^{\text {hi }}$.

Flow cytometry. All antibody details can be found in Supplemental Table 1.

Hematopoietic stem and progenitor cells. BM cells were obtained from femurs and tibias and stained with a cocktail of antibodies before analysis by flow cytometry as previously described $(10,48,70)$. Briefly, lineage-committed cells were identified as CD45R, CD19, CD11b, CD3e, TER-119, CD2, CD8, CD4, and Ly6-C/G, all FITC positive, with antibodies against $\mathrm{Sca} 1$ and cKit to identify progenitor cell populations and HSPCs (Lineage ${ }^{-}, \mathrm{Sca}^{+}, \mathrm{cKit}^{+}$) and with antibodies against CD16/ CD32 (Fc $\gamma$ RII/III) and CD34 to separate CMPs (Lineage-, Sca1-, $\mathrm{cKit}^{+}$, CD34 ${ }^{\text {int }}$, Fc $\gamma$ RII/III ${ }^{\text {int }}$ ), MEPs (Lineage ${ }^{-}, \mathrm{Sca}^{-}, \mathrm{cKit}^{+}, \mathrm{CD} 34^{\text {lo }}$, Fc $\gamma \mathrm{RII} /$ $\mathrm{III}^{\mathrm{lo}}$ ), and MkPs (Lineage ${ }^{-}, \mathrm{Sca}^{-}, \mathrm{cKit}^{+}, \mathrm{CD} 34^{\text {int }}, \mathrm{Fc} \gamma \mathrm{RII} / \mathrm{III}{ }^{\mathrm{int}}, \mathrm{CD} 41^{+}$). Cell cycle was quantified using DAPI (Sigma-Aldrich). The antibody to c-MPL was a gift from W. Tong (University of Pennsylvania, Philadelphia, Pennsylvania, USA) (71).

Neutrophil- and monocyte-platelet aggregates. Blood was collected through the tail vein into EDTA-lined tubes on ice to prevent leukocyte activation. Red blood cells were lysed, and the washed cells were then stained with CD45, CD115, Gr1 (Ly6-C/G), CD11b, and CD41 at 1:200 dilution for 30 minutes on ice. The cells were carefully washed, resuspended in FACS buffer, and run on an LSRII flow cytometer to detect leukocyte-platelet interactions and leukocyte activation. Viable cells were selected on the basis of forward and side scatter characteristics, and then $\mathrm{CD} 45^{+}$leukocytes were selected. Ly6- $\mathrm{C}^{\text {hi }}$ monocyte-platelet aggregates were identified as $\mathrm{CD} 115^{+} \mathrm{Gr} 1^{\text {hi }}\left(\mathrm{Ly} 6-\mathrm{C}^{\text {hi }}\right)$ and $\mathrm{CD} 41^{+}$. Neutrophil-platelet aggregates were identified as $\mathrm{CD} 115^{-} \mathrm{Gr}^{+}\left(\mathrm{Ly} 6-\mathrm{G}^{+}\right)$ and $\mathrm{CD} 41^{+}$. Platelet-dependent activation of Ly6- $\mathrm{C}^{\text {hi }}$ monocytes and neutrophils was measured by CD11b expression (geo-mean fluorescence intensity [MFI]).

Neutrophil depletion. Neutrophils were depleted in WT and diabetic (STZ) mice after the onset of diabetes for 4 weeks, by i.p. injection of the neutrophil-specific anti-Ly6-G antibody (clone 1A8; $1 \mathrm{mg} /$ injection) every 3 days. This antibody is specific to neutrophils and does not affect monocytes (72).

$B M$-derived macrophages. BM-derived macrophages (BMDMs) were generated from the BM of hind limb bones of WT C57BL/6 mice. Following 7 days of culture in RPMI media containing 15\% FBS and 20\% L cell-conditioned media, BMDMs were changed into fresh RPMI media containing 5\% FBS and 2\% BSA before treatment in high-glucose media ( $25 \mathrm{mmol} / \mathrm{l})$.

RNA isolation, cDNA synthesis, and quantitative real time PCR. Total RNA from cells was extracted using QIAGEN RNeasy Mini or Micro kits and cDNA synthesized using Superscript Vilo (Invitrogen, Thermo Fisher Scientific). Quantitative real time PCR was monitored in real time with an Mx3000 sequence detection system (Stratagene) using SYBR Green PCR Core Reagents (Agilent Technologies) and normalized to $m 36 b 4$.

BM megakaryocytes. Bones were fixed in $4 \%$ paraformaldehyde for 24 hours and then decalcified with EDTA solution and sectioned. $\mathrm{H} \& \mathrm{E}$ staining was performed. Megakaryocytes were identified as large multinucleated cells within the marrow.

Atherosclerosis study. Apoe $e^{-/}$mice were fed a chow diet, and a portion of mice were made diabetic with STZ. A group of STZ mice were treated with ABR-215757 (10 mg/kg/d in drinking water). After 12 weeks of diabetes the mice were sacrificed for atherosclerotic lesion analysis.

Total cholesterol. Cholesterol levels in the plasma were quantified using the Wako total cholesterol kit as previously described (6).

Lesion analysis. Hearts were dissected after the mice were perfused with saline and frozen in OCT compound. Serial 6- $\mu \mathrm{m}$ sections of the proximal aorta were prepared. H\&E staining was performed as previously described (73). Lipid content was assessed by Oil Red $\mathrm{O}$ staining, and macrophage content was quantified by staining for CD68 (6). Acellular regions were identified as nuclei-free regions, and collagen was quantified by staining with Picrosirius red and capturing of images under polarized light. Images were captured on an Olympus BX43 microscope, and quantification of all images was performed using Adobe Photoshop CS5.

Aortic arch lipid analysis. Lipid content in the aortic arch was measured by en face analysis. Dissected aortas were fixed in paraformaldehyde, and before staining, all fat and connective tissue was removed from the outer layers of the vessel. The aorta was cut longitudinally and stained with Oil Red O followed by washing and mounting on a silicone-coated dish. Aortas were viewed on an Olympus SZX10 and captured using Q-Capture Pro 7 (QImaging) software. Quantification of Oil Red O staining was performed off-line using Adobe Photoshop CS5.

Human study. The human study was conducted at New York University Langone Medical Center. Exclusion criteria were use of other antiplatelet therapy including use of anticoagulant and NSAIDs within 
72 hours, platelet count less than $100 \times 10^{9}$ per liter or greater than 500 $\times 10^{9}$ per liter, hemoglobin less than 9 , any hemorrhagic diathesis, or severe kidney disease. Peripheral blood was drawn from subjects and the first $5 \mathrm{ml}$ was discarded, and the remaining blood was collected into tubes containing $3.2 \%$ sodium citrate for subsequent determination of platelet activity. Samples were processed within 30 minutes of collection. Flow cytometry was used to analyze monocyte-platelet aggregates in whole blood as previously described $(42,74)$. Reticulated platelets were measured with a previously described flow cytometry assay (75).

Statistics. Data are presented as mean \pm SEM (unless stated otherwise) and were analyzed using the 2-tailed Student's $t$ test or 1-way ANOVA followed by Newman-Keuls post hoc test. $P$ less than 0.05 was considered significant. All tests were performed using Prism software (GraphPad Software Inc.).

Study approval. Animal experiments were approved by the Alfred Medical Research Education Precinct Animal Ethics Committee, Melbourne, Australia, and conducted in accordance with the National Health and Medical Research Council (NHMRC) of Australia Guidelines for Animal Experimentation. The total-body S100a9-/ experiments were conducted at New York University and were approved by the New York University animal ethics committee.

The human studies were conducted in accordance with policies of the New York University Langone Medical Center Institutional Review Board. All patients provided consent prior to participation in the study. From ongoing studies, we identified subjects on aspirin in an ongoing registry and ongoing studies (NCT02106429 and NCT01897103, ClinicalTrials.gov) measuring platelet activity.

Detailed methods are available in Supplemental Methods.

\section{Author contributions}

MJK, MKSL, JSB, IJG, PRN, and AJM designed and conducted experiments, analyzed results, and wrote the manuscript. AAS, DD, TJB, EM, DB, SH, HLK, MF, AW, and NMJH conducted experiments and analyzed data. MAF, EW, EAF, JCD, and MEC provided resources and intellectual input. All authors edited the manuscript.

\section{Acknowledgments}

This work was supported by NIH grants (HL092969 and DK095684) to IJG, an NIH grant (R0OHL122505) and startup funds (from the University of Alabama at Birmingham) to PRN, and NHMRC grants (APP1083138 and APP1106154) to AJM and EW. TJB and EAF were supported by NIH grant DK095684. MJK is a Russell Berrie Foundation Scholar in Diabetes Research from the Naomi Berrie Diabetes Centre. MAF is a Senior Principal Research Fellow of the NHMRC (APP1021168). AJM is supported by a career development fellowship from the NHMRC (APP1085752), a future leader fellowship from the National Heart Foundation (100440), and a Viertel award from Diabetes Australia Research Trust.

Address correspondence to: Andrew J. Murphy, Haematopoiesis and Leukocyte Biology, Baker Heart and Diabetes Institute, 75 Commercial Road, Melbourne, Victoria 3004, Australia. Phone: 61.3.8532.1292; E-mail: andrew.murphy@bakeridi.edu.au. Or to: Prabhakara R. Nagareddy, Department of Nutrition Sciences, University of Alabama at Birmingham, 1825 University Boulevard, Shelby Building, Room 303, Birmingham, Alabama 35294, USA. Phone: 205.934.6754; E-mail:pnreddy@uab.edu.
1. Haffner SM, Lehto S, Rönnemaa T, Pyörälä K, Laakso M. Mortality from coronary heart disease in subjects with type 2 diabetes and in nondiabetic subjects with and without prior myocardial infarction. N Engl JMed.1998;339(4):229-234.

2. Laakso M. Cardiovascular disease in type 2 diabetes from population to man to mechanisms: the Kelly West Award Lecture 2008. Diabetes Care. 2010;33(2):442-449.

3. Shah $\mathrm{AD}$, et al. Type 2 diabetes and incidence of cardiovascular diseases: a cohort study in 1.9 million people. Lancet Diabetes Endocrinol. 2015;3(2):105-113.

4. Lüscher TF, Creager MA, Beckman JA, Cosentino F. Diabetes and vascular disease: pathophysiology, clinical consequences, and medical therapy: part II. Circulation. 2003;108(13):1655-1661.

5. Pasterkamp G. Methods of accelerated atherosclerosis in diabetic patients. Heart. 2013;99(10):743-749.

6. Nagareddy PR, et al. Hyperglycemia promotes myelopoiesis and impairs the resolution of atherosclerosis. Cell Metab. 2013;17(5):695-708.

7. Sokunbi DO, Wadhwa NK, Suh H. Vascular disease outcome and thrombocytosis in diabetic and nondiabetic end-stage renal disease patients on peritoneal dialysis. Adv Perit Dial. 1994;10:77-80.

8. Huo Y, et al. Circulating activated platelets exacerbate atherosclerosis in mice deficient in apolipoprotein E. Nat Med. 2003;9(1):61-67.

9. Koenen RR, et al. Disrupting functional interactions between platelet chemokines inhibits atherosclerosis in hyperlipidemic mice. Nat Med.
2009;15(1):97-103.

10. Murphy AJ, et al. Cholesterol efflux in megakaryocyte progenitors suppresses platelet production and thrombocytosis. Nat Med. 2013;19(5):586-594.

11. Martin JF, Kristensen SD, Mathur A, Grove EL, Choudry FA. The causal role of megakaryocyteplatelet hyperactivity in acute coronary syndromes. Nat Rev Cardiol. 2012;9(11):658-670.

12. [No authors listed]. Collaborative overview of randomised trials of antiplatelet therapy - I: Prevention of death, myocardial infarction, stroke by prolonged antiplatelet therapy in various categories of patients. Antiplatelet Trialists' Collaboration. BMJ. 1994;308(6921):81-106.

13. Angiolillo DJ. Antiplatelet therapy in diabetes: efficacy and limitations of current treatment strategies and future directions. Diabetes Care. 2009;32(4):531-540.

14. Guthikonda S, et al. Role of reticulated platelets and platelet size heterogeneity on platelet activity after dual antiplatelet therapy with aspirin and clopidogrel in patients with stable coronary artery disease. J Am Coll Cardiol. 2008;52(9):743-749.

15. Neergaard-Petersen S, Hvas AM, Kristensen SD Grove EL. Platelets and antiplatelet therapy in patients with coronary artery disease and diabetes. Semin Thromb Hemost. 2016;42(3):234-241.

16. Papanas N, et al. Mean platelet volume in patients with type 2 diabetes mellitus. Platelets. 2004;15(8):475-478.

17. Muscari A, et al. Determinants of mean platelet volume (MPV) in an elderly population: relevance of body fat, blood glucose and ischaemic electrocardiographic changes. Thromb Haemost. 2008;99(6):1079-1084.

18. Guthikonda S, et al. Reticulated platelets and uninhibited COX-1 and COX-2 decrease the antiplatelet effects of aspirin. J Thromb Haemost. 2007;5(3):490-496.

19. Hekimsoy Z, Payzin B, Ornek T, Kandoğan G. Mean platelet volume in type 2 diabetic patients. J Diabetes Complicat. 2004;18(3):173-176.

20. Shah B, Valdes V, Nardi MA, Hu L, Schrem E, Berger JS. Mean platelet volume reproducibility and association with platelet activity and anti-platelet therapy. Platelets. 2014;25(3):188-192.

21. Tschoepe D, et al. Large platelets circulate in an activated state in diabetes mellitus. Semin Thromb Hemost. 1991;17(4):433-438.

22. Vaduganathan $M$, et al. Platelet reactivity and response to aspirin in subjects with the metabolic syndrome. Am Heart J. 2008;156(5):1002.e1-1002.e7.

23. Karpatkin S. Heterogeneity of human platelets. II. Functional evidence suggestive of young and old platelets. JClin Invest. 1969;48(6):1083-1087.

24. Kleiman NS. Are immature platelets growing up?: Toward a new marker of antiplatelet drug resistance. J Am Coll Cardiol. 2016;68(3):294-296.

25. Stratz C, et al. Comparison of immature platelet count to established predictors of platelet reactivity during thienopyridine therapy. J Am Coll Cardiol. 2016;68(3):286-293.

26. Junt $\mathrm{T}$, et al. Dynamic visualization of throm- 
bopoiesis within bone marrow. Science. 2007;317(5845):1767-1770.

27. Schafer AI. Thrombocytosis. N Engl J Med. 2004;350(12):1211-1219.

28. Sungaran R, Markovic B, Chong BH. Localization and regulation of thrombopoietin $\mathrm{mRNa}$ expression in human kidney, liver, bone marrow, and spleen using in situ hybridization. Blood. 1997;89(1):101-107.

29. Wolber EM, Fandrey J, Frackowski U, Jelkmann W. Hepatic thrombopoietin mRNA is increased in acute inflammation. Thromb Haemost. 2001;86(6):1421-1424.

30. Kaser A, et al. Interleukin-6 stimulates thrombopoiesis through thrombopoietin: role in inflammatory thrombocytosis. Blood. 2001;98(9):2720-2725.

31. Burmester H, Wolber EM, Freitag P, Fandrey J, Jelkmann $\mathrm{W}$. Thrombopoietin production in wild-type and interleukin- 6 knockout mice with acute inflammation. JInterferon Cytokine Res. 2005;25(7):407-413.

32. Gieger $\mathrm{C}$, et al. New gene functions in megakaryopoiesis and platelet formation. Nature. 2011;480(7376):201-208.

33. Koppikar P, et al. Efficacy of the JAK2 inhibitor INCB16562 in a murine model of MPLW515L-induced thrombocytosis and myelofibrosis. Blood. 2010;115(14):2919-2927.

34. Pikman Y, et al. MPLW515L is a novel somatic activating mutation in myelofibrosis with myeloid metaplasia. PLoS Med.2006;3(7):e270.

35. Eriksson U, Ewald U, Tuvemo T. Increased platelet volume in manifest diabetic rats. Ups JMed Sci. 1983;88(1):17-23.

36. Bacon CM, Tortolani PJ, Shimosaka A, Rees RC, Longo DL, O'Shea JJ. Thrombopoietin (TPO) induces tyrosine phosphorylation and activation of STAT5 and STAT3. FEBS Lett. 1995;370(1-2):63-68.

37. Wong CH, Jenne CN, Petri B, Chrobok NL, Kubes P. Nucleation of platelets with blood-borne pathogens on Kupffer cells precedes other innate immunity and contributes to bacterial clearance. Nat Immunol. 2013;14(8):785-792.

38. Scott CL, et al. Bone marrow-derived monocytes give rise to self-renewing and fully differentiated Kupffer cells. Nat Commun. 2016;7:10321.

39. Klein I, et al. Kupffer cell heterogeneity: functional properties of bone marrow derived and sessile hepatic macrophages. Blood. 2007;110(12):4077-4085.

40. Shah B, Sha D, Xie D, Mohler ER, Berger JS. The relationship between diabetes, metabolic syndrome, and platelet activity as measured by mean platelet volume: the National Health And Nutrition Examination Survey, 1999-2004. Diabetes Care. 2012;35(5):1074-1078.

41. Björk P, et al. Identification of human S100A9 as a novel target for treatment of autoimmune disease via binding to quinoline-3-carboxamides. PLoS Biol. 2009;7(4):e97.

42. Michelson AD, Barnard MR, Krueger LA, Valeri CR, Furman MI. Circulating monocyte-platelet aggregates are a more sensitive marker of in vivo platelet activation than platelet surface P-selectin: studies in baboons, human coronary inter- vention, and human acute myocardial infarction. Circulation. 2001;104(13):1533-1537.

43. Hiro T, et al. Diabetes mellitus is a major negative determinant of coronary plaque regression during statin therapy in patients with acute coronary syndrome - serial intravascular ultrasound observations from the Japan Assessment of Pitavastatin and Atorvastatin in Acute Coronary Syndrome Trial (the JAPAN-ACS Trial). Circ J. 2010;74(6):1165-1174.

44. Bayraktar M, Dündar S, Kirazli S, Teletar F. Platelet factor $4, \beta$-thromboglobulin and thrombospondin levels in type I diabetes mellitus patients. JInt Med Res. 1994;22(2):90-94.

45. Roy MS, Podgor MJ, Rick ME. Plasma fibrinopeptide A, beta-thromboglobulin, and platelet factor 4 in diabetic retinopathy. Invest Ophthalmol Vis Sci. 1988;29(6):856-860.

46. Slavka G, et al. Mean platelet volume may represent a predictive parameter for overall vascular mortality and ischemic heart disease. Arterioscler Thromb Vasc Biol. 2011;31(5):1215-1218.

47. Nicolucci A, Standl E. Antiplatelet therapy for every diabetic person? Diabetes Care. 2011;34 Suppl 2:S150-S154.

48. Murphy AJ, et al. Deficiency of ATP-binding cassette transporter B6 in megakaryocyte progenitors accelerates atherosclerosis in mice. Arterioscler Thromb Vasc Biol. 2014;34(4):751-758.

49. Murphy AJ, Tall AR. Disordered haematopoiesis and athero-thrombosis. Eur Heart $J$. 2016;37(14):1113-1121.

50. Maugeri $\mathrm{N}$, et al. Clearance of circulating activated platelets in polycythemia vera and essential thrombocythemia. Blood. 2011;118(12):3359-3366

51. Grove EL, Hvas AM, Mortensen SB, Larsen SB, Kristensen SD. Effect of platelet turnover on whole blood platelet aggregation in patients with coronary artery disease. J Thromb Haemost. 2011;9(1):185-191.

52. Stone RL, et al. Paraneoplastic thrombocytosis in ovarian cancer. NEnglJMed.2012;366(7):610-618.

53. Jenne $C N$, Kubes P. Immune surveillance by the liver. Nat Immunol. 2013;14(10):996-1006.

54. Gregory SH, Wing EJ, Danowski KL, van Rooijen N, Dyer KF, Tweardy DJ. IL-6 produced by Kupffer cells induces STAT protein activation in hepatocytes early during the course of systemic listerial infections. J Immunol. 1998;160(12):6056-6061.

55. Kubes P, Mehal WZ. Sterile inflammation in the liver. Gastroenterology. 2012;143(5):1158-1172.

56. Fuentes E, Rojas A, Palomo I. Role of multiligand/RAGE axis in platelet activation. Thromb Res. 2014;133(3):308-314.

57. Nagareddy PR, et al. Adipose tissue macrophages promote myelopoiesis and monocytosis in obesity. Cell Metab. 2014;19(5):821-835

58. Croce $\mathrm{K}$, et al. Myeloid-related protein- $8 / 14$ is critical for the biological response to vascular injury. Circulation. 2009;120(5):427-436.

59. Wang Y, et al. Platelet-derived S100 family member myeloid-related protein-14 regulates thrombosis. J Clin Invest. 2014;124(5):2160-2171.

60. Dindar S, Cinemre H, Sengul E, Annakkaya
AN. Mean platelet volume is associated with glycaemic control and retinopathy in patients with type 2 diabetes mellitus. West Indian Med J . 2013;62(6):519-523.

61. Demirtunc R, Duman D, Basar M, Bilgi M, Teomete $\mathrm{M}, \mathrm{Garip} \mathrm{T}$. The relationship between glycemic control and platelet activity in type 2 diabetes mellitus. JDiabetes Complicat. 2009;23(2):89-94.

62. Node K, Inoue T. Postprandial hyperglycemia as an etiological factor in vascular failure. Cardiovasc Diabetol. 2009;8:23.

63. Leiter LA, et al. Postprandial glucose regulation: new data and new implications. Clin Ther. 2005;27(suppl B):S42-S56.

64. Ceriello A. Postprandial hyperglycemia and diabetes complications: is it time to treat? Diabetes. 2005;54(1):1-7.

65. Cavalot F, et al. Postprandial blood glucose is a stronger predictor of cardiovascular events than fasting blood glucose in type 2 diabetes mellitus, particularly in women: lessons from the San Luigi Gonzaga Diabetes Study. JClin Endocrinol Metab. 2006;91(3):813-819.

66. Aryangat AV, Gerich JE. Type 2 diabetes: postprandial hyperglycemia and increased cardiovascular risk. Vasc Health Risk Manag. 2010;6:145-155.

67. Zinman B, et al. Empagliflozin, cardiovascular outcomes, and mortality in type 2 diabetes. N Engl J Med. 2015;373(22):2117-2128.

68. Bengtsson AA, et al. Pharmacokinetics, tolerability, and preliminary efficacy of paquinimod (ABR-215757), a new quinoline-3-carboxamide derivative: studies in lupus-prone mice and a multicenter, randomized, double-blind, placebo-controlled, repeat-dose, dose-ranging study in patients with systemic lupus erythematosus. Arthritis Rheum. 2012;64(5):1579-1588.

69. Manitz MP, et al. Loss of S100A9 (MRP14) results in reduced interleukin-8-induced CD11b surface expression, a polarized microfilament system, and diminished responsiveness to chemoattractants in vitro. Mol Cell Biol. 2003;23(3):1034-1043.

70. Murphy AJ, et al. ApoE regulates hematopoietic stem cell proliferation, monocytosis, and monocyte accumulation in atherosclerotic lesions in mice. JClin Invest. 2011;121(10):4138-4149.

71. Tong W, Ibarra YM, Lodish HF. Signals emanating from the membrane proximal region of the thrombopoietin receptor (mpl) support hematopoietic stem cell self-renewal. Exp Hematol. 2007;35(9):1447-1455.

72. Daley JM, Thomay AA, Connolly MD, Reichner JS, Albina JE. Use of Ly6G-specific monoclonal antibody to deplete neutrophils in mice. J Leukoc Biol. 2008;83(1):64-70.

73. Renard CB, et al. Diabetes and diabetes-associated lipid abnormalities have distinct effects on initiation and progression of atherosclerotic lesions. JClin Invest. 2004;114(5):659-668.

74. Shah B, et al. Comparison of platelet activity measurements by use of arterial and venous blood sampling. J Thromb Haemost. 2013;11(10):1922-1924.

75. Michelson AD, Barnard MR, Krueger LA, Frelinger AL, Furman MI. Evaluation of platelet function by flow cytometry. Methods. 2000;21(3):259-270. 